Supporting Information

\title{
Arrays of Microscale Linear Ridges with Self-Cleaning Functionality for the Oxygen Evolution Reaction
}

Audrey K. Taylor, Tiffany Mou, Ana Sonea, Jiayue Chen, Brenden B. Yee, and Byron D. Gates *

\author{
Department of Chemistry \\ Simon Fraser University \\ 8888 University Drive \\ Burnaby, BC V5A 1S6 (Canada) \\ Telephone Number: (778) 782-8066 \\ Fax Number: (778) 782-3765 \\ Email Address: * bgates@sfu.ca
}

This research was supported in part by the Natural Sciences and Engineering Research Council of Canada (NSERC; Grant No. RGPIN-2020-06522), the Canada Research Chairs Program (B.D. Gates, Grant No. 950-215846), the Engineered Nickel Catalysts for Electrochemical Clean Energy project administered from Queen's University (Grant No. RGPNM 477963-2015) from the NSERC of Canada Discovery Frontiers Program, and CMC Microsystems (MNT Financial Assistance, Grant No. 5355). This work made use of the 4D LABS (www.4dlabs.ca) and the Center for Soft Materials shared facilities supported by the Canada Foundation for Innovation (CFI), British Columbia Knowledge Development Fund (BCKDF), Western Economic Diversification Canada, and Simon Fraser University. 


\section{Table of Contents}

\section{Section S1.}

High magnification scanning electron microscopy (SEM) image of a linear ridge.........S4

Representative SEM images of a planar electrode................................. 5

Stitched optical microscopy image of a patterned electrode.........................S6

Plot of the surface area percent increase for the patterned electrodes....................S7

Electrochemical activation by cyclic voltammetry $(\mathrm{CV})$ techniques....................S

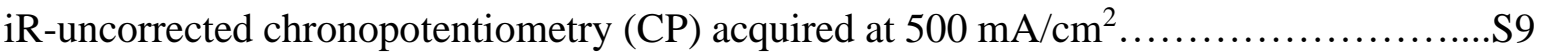

Histograms of the iR-corrected voltage responses acquired at $500 \mathrm{~mA} / \mathrm{cm}^{2} \ldots \ldots \ldots \ldots . . . \mathrm{S} 10$

Representative CP plots acquired at $500 \mathrm{~mA} / \mathrm{cm}^{2}$ over a short time-scale...............S11

A series of chronoamperometry (CA) measurements for the replicate 10-200 electrodes S12

A series of CA measurements for the replicate 10-100 electrodes.....................S13

A series of CA measurements for the replicate 10-50 electrodes.......................S14

A series of CA measurements for the replicate 10-25 electrodes......................S15

A series of CA measurements for the replicate 10-10 electrodes.....................S16

A series of CA measurements for the replicate planar electrodes.....................S17

Summary of the mean current densities for all electrode types.......................S18

Histograms of the CA measurements for all representative electrodes..................S19

Oxygen evolution reaction (OER) performance by linear sweep voltammetry (LSV).....S20

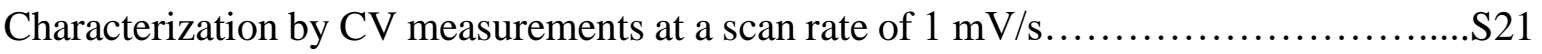

A table of the electrochemically active surface area $\left(A_{\text {ecsa }}\right)$ values...................... 22

Representative frames from the video footage for the 10-200 electrode..............S23

Representative frames from the video footage for the 10-100 electrode................S24

Representative frames from the video footage for the $10-50$ electrode................S25

Representative frames from the video footage for the 10-25 electrode.................S26

Representative frames from the video footage for the 10-10 electrode................S27

Representative frames from the video footage for the planar electrode.................S28 


\section{Section S2.}

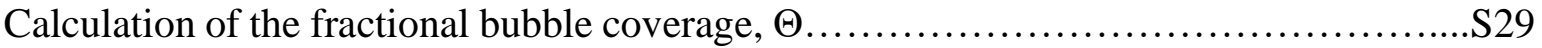

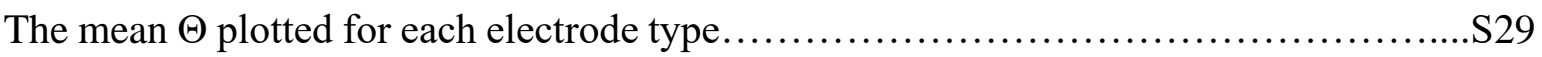

Plot of the mean bubble departure diameters for each electrode type ...................S30

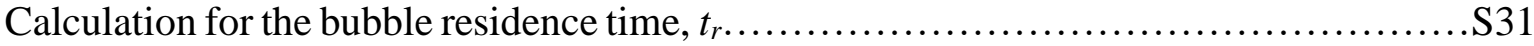

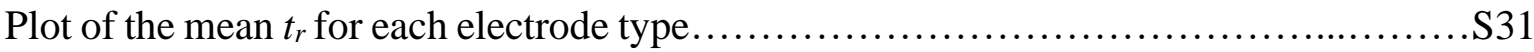

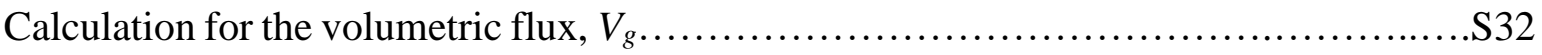

Calculations for the bubble related overpotentials...................................S33

Plot of the activation, ohmic, and concentration overpotentials.........................S34

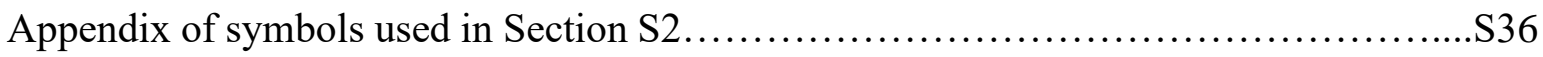

\section{Section S3.}

Representative SEM images acquired after electrochemical measurements...............S37

A plot of the mean gas bubble contact angles (BCAs) for each electrode type.............S38

A table of BCA values for each electrode type.......................................... 39

A photograph of the BCA measurement set-up......................................... 440

\section{References}




\section{Section S1.}

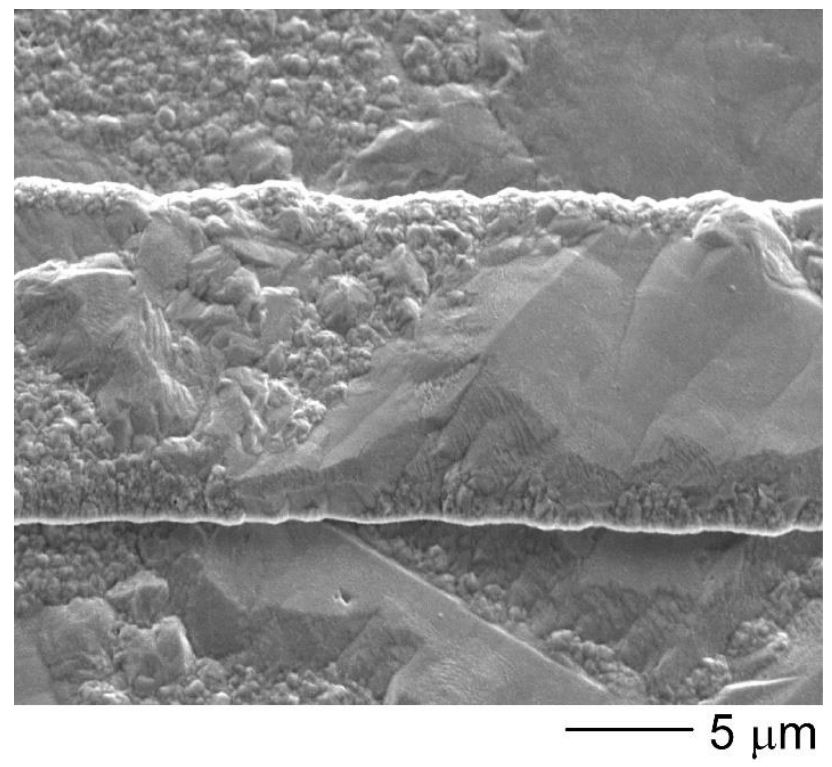

Figure S1. Representative high magnification scanning electron microscopy (SEM) image of a linear ridge of electrodeposited nickel (Ni). This SEM image was acquired at a $35^{\circ}$ tilt. 


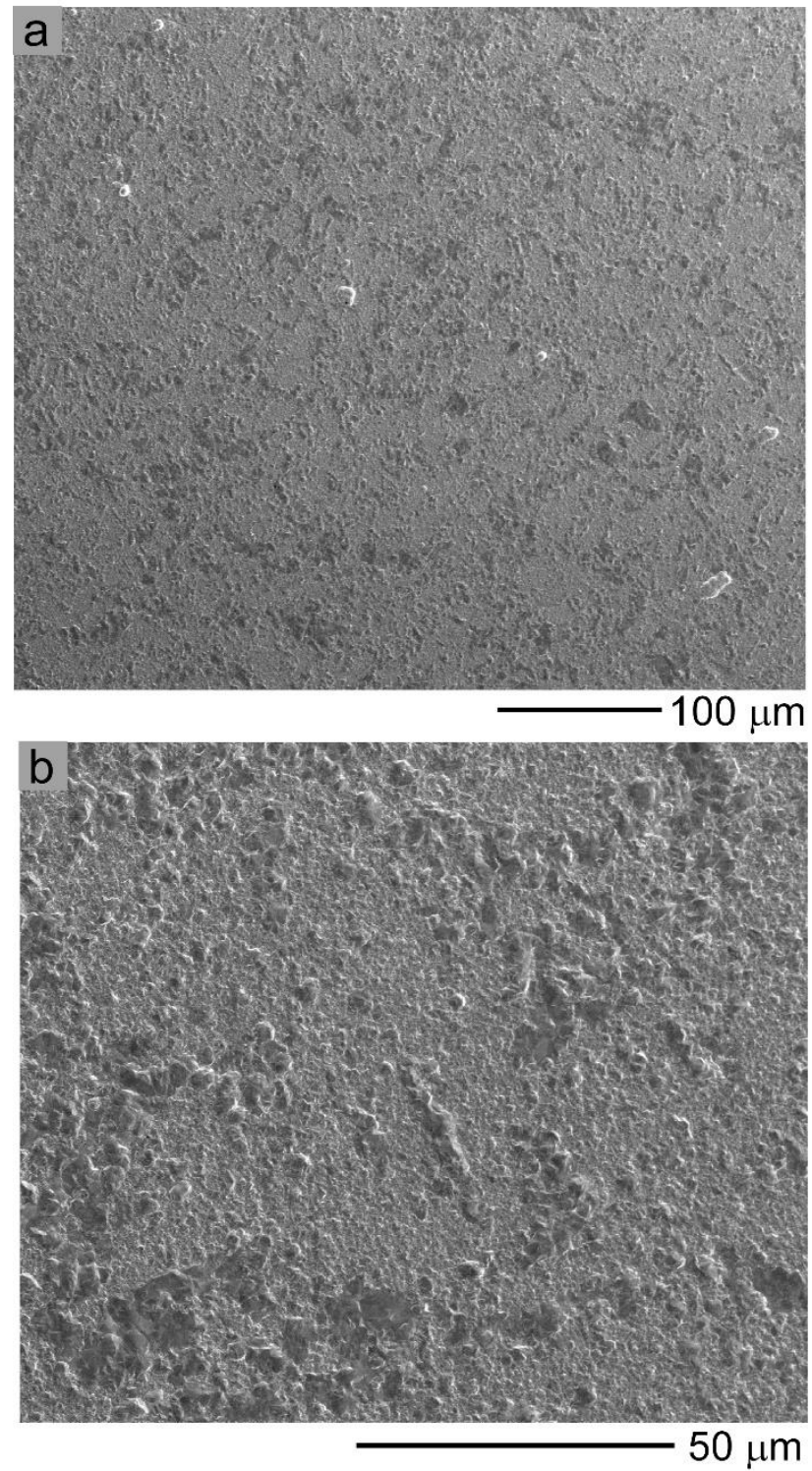

Figure S2. Representative SEM images of the planar electrodeposited Ni surfaces at (a) low and (b) high magnifications. These images were acquired prior to the electrochemical measurements. 


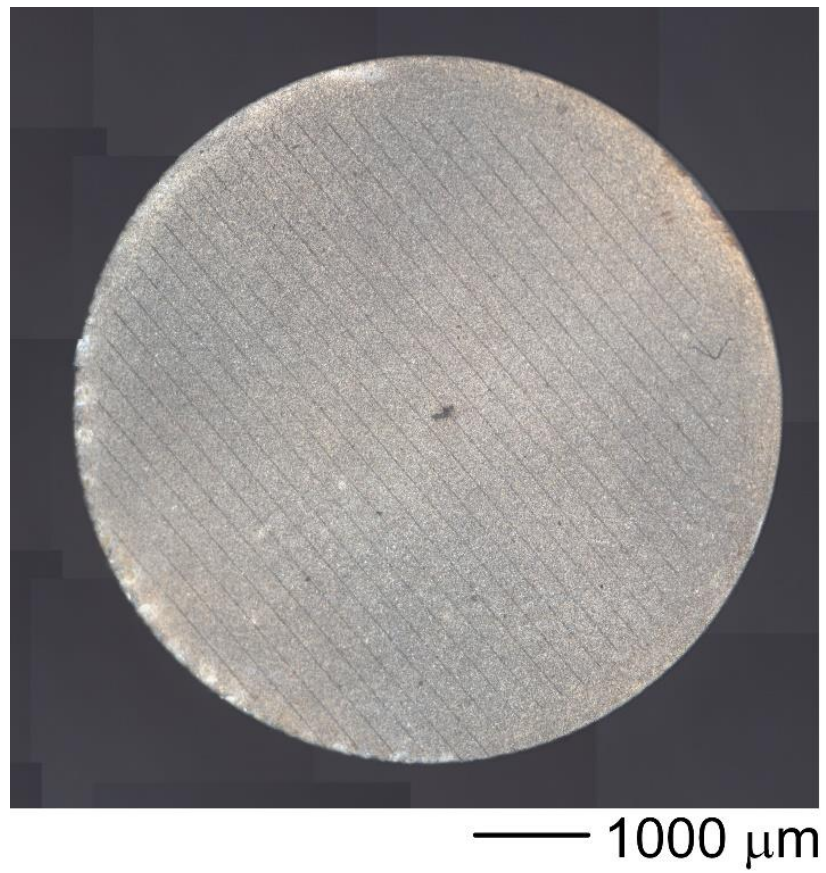

Figure S3. Representative stitched optical microscopy image of the patterned interface on the working electrode with an array of 10-200 linear ridges. 


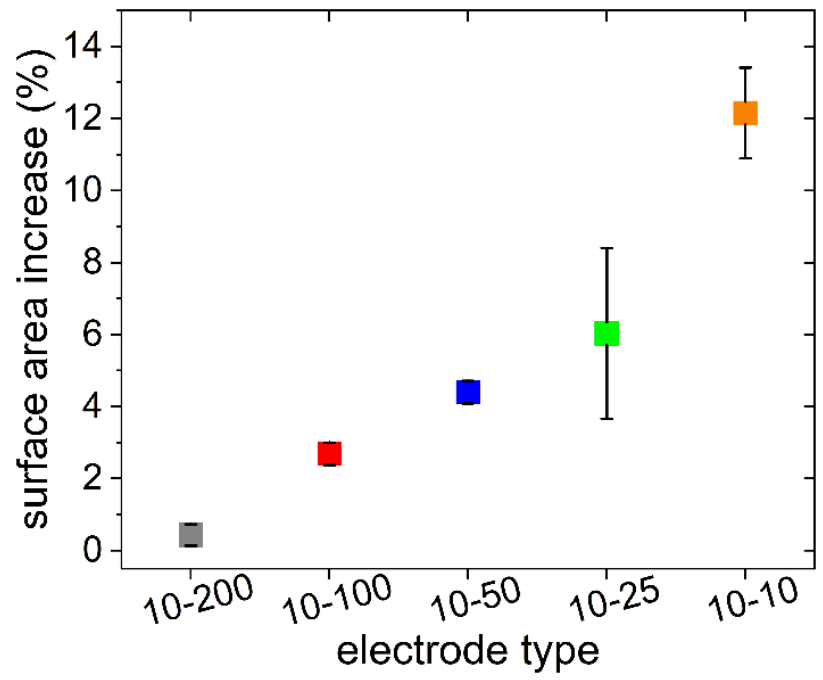

Figure S4. The measured surface area increases relative to a planar electrode due to the presence of the arrays of linear microscale ridges prepared by photolithography and $\mathrm{Ni}$ electrodeposition on the electrode surfaces. The error bars represent one standard deviation from the calculated mean values. Each of the calculated mean values were obtained from a sample set of three individual electrodes. Four separate sets of measurements of the ridge heights were acquired for each electrode. Three individual electrodes were analyzed for each type of patterned electrode. A minimum of twelve measurements were used to derive each mean value. 


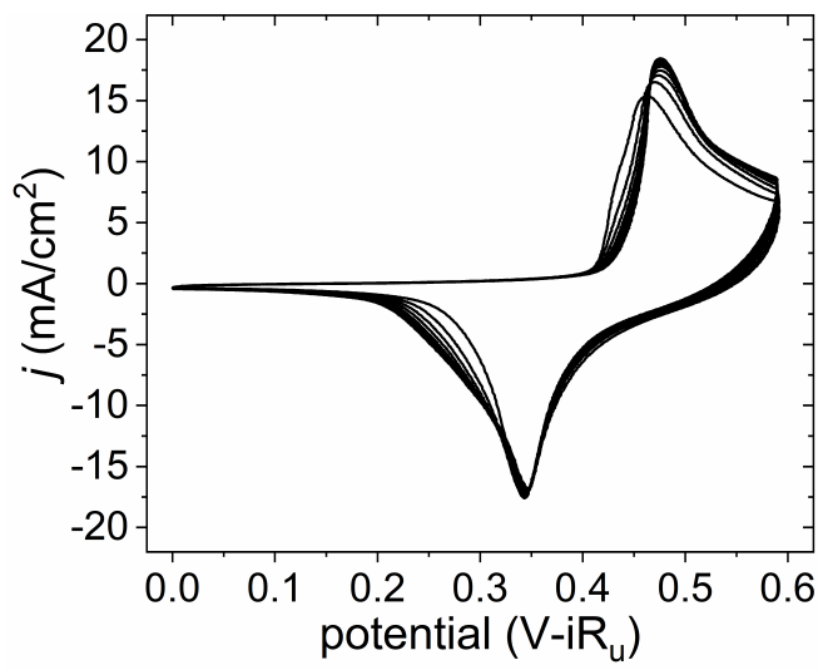

Figure S5. Representative electrochemical activation of the $\mathrm{Ni}$ electrodes using a cyclic voltammetry (CV) technique as depicted here for an array of 10-100 linear ridges. The data is plotted for every 5 scans between the $5^{\text {th }}$ and $45^{\text {th }} \mathrm{CV}$ profile. A total of 50 scans were performed in purified $1 \mathrm{M} \mathrm{KOH}$ at a scan rate of $100 \mathrm{mV} / \mathrm{s}$ for electrochemically activating each of the electrodes. 


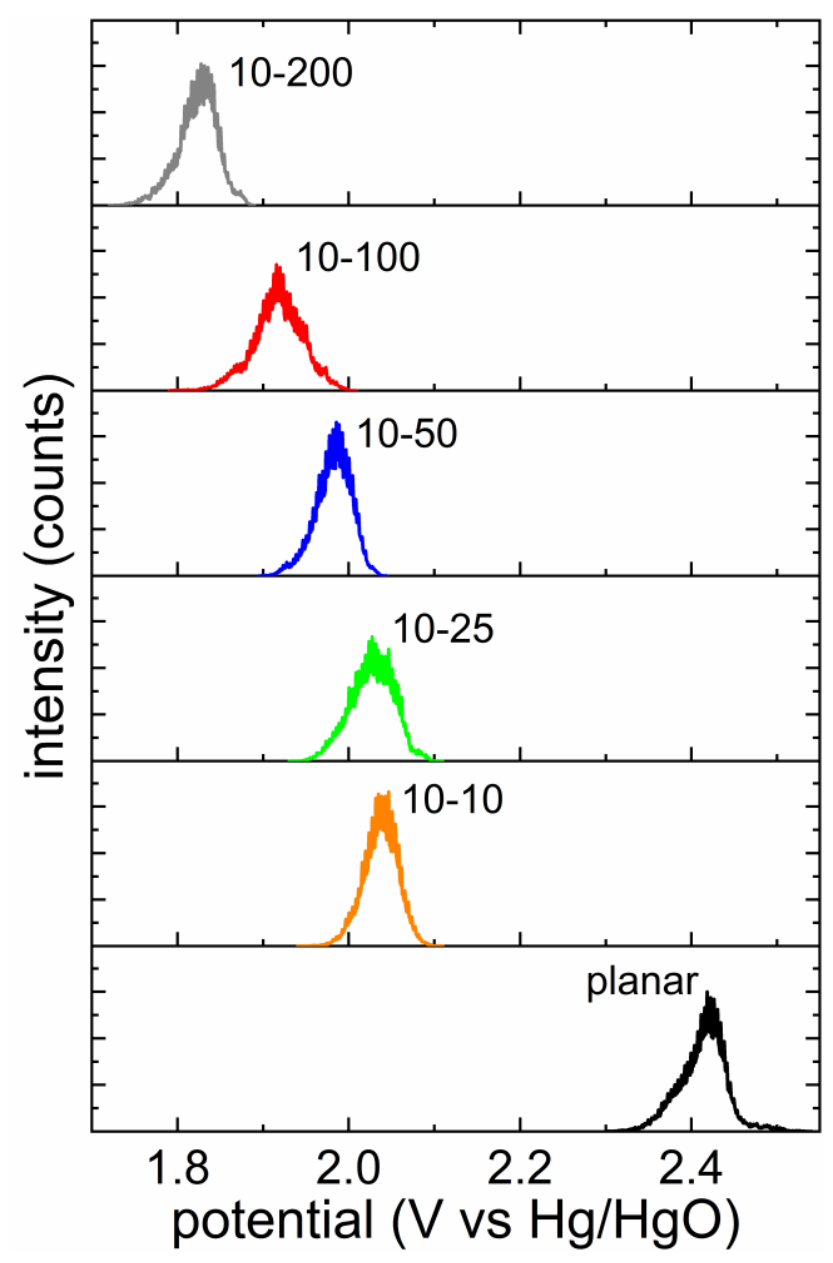

Figure S6. Representative histograms of the iR-uncorrected chronopotentiometry (CP) results for each type of Ni electrode acquired at $500 \mathrm{~mA} / \mathrm{cm}^{2}$ in purified $1 \mathrm{M} \mathrm{KOH}$. These histograms depict the distribution of the potentials recorded for the last $1200 \mathrm{~s}$ of each set of measurements. 

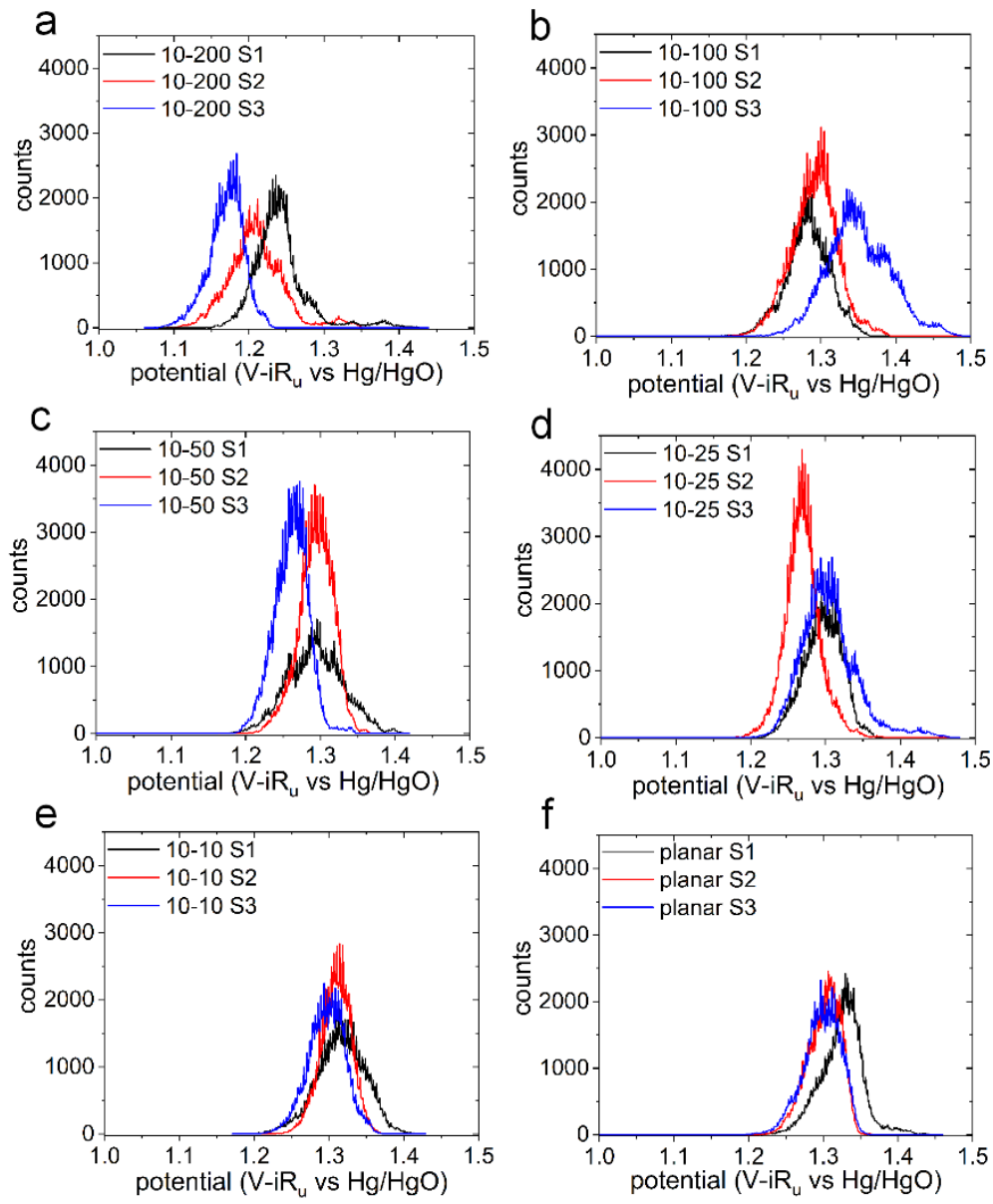

Figure S7. Histograms of the iR-corrected voltage responses for each type of electrode (i.e., with microscale Ni patterns or planar Ni) recorded during the $\mathrm{CP}$ experiments with a current density of $500 \mathrm{~mA} / \mathrm{cm}^{2}$. The responses for three replicate samples are shown for each type of electrode: (a) 10-200; (b) 10-100; (c) 10-50; (d) 10-25; (e) 10-10; and (f) planar electrodes. 


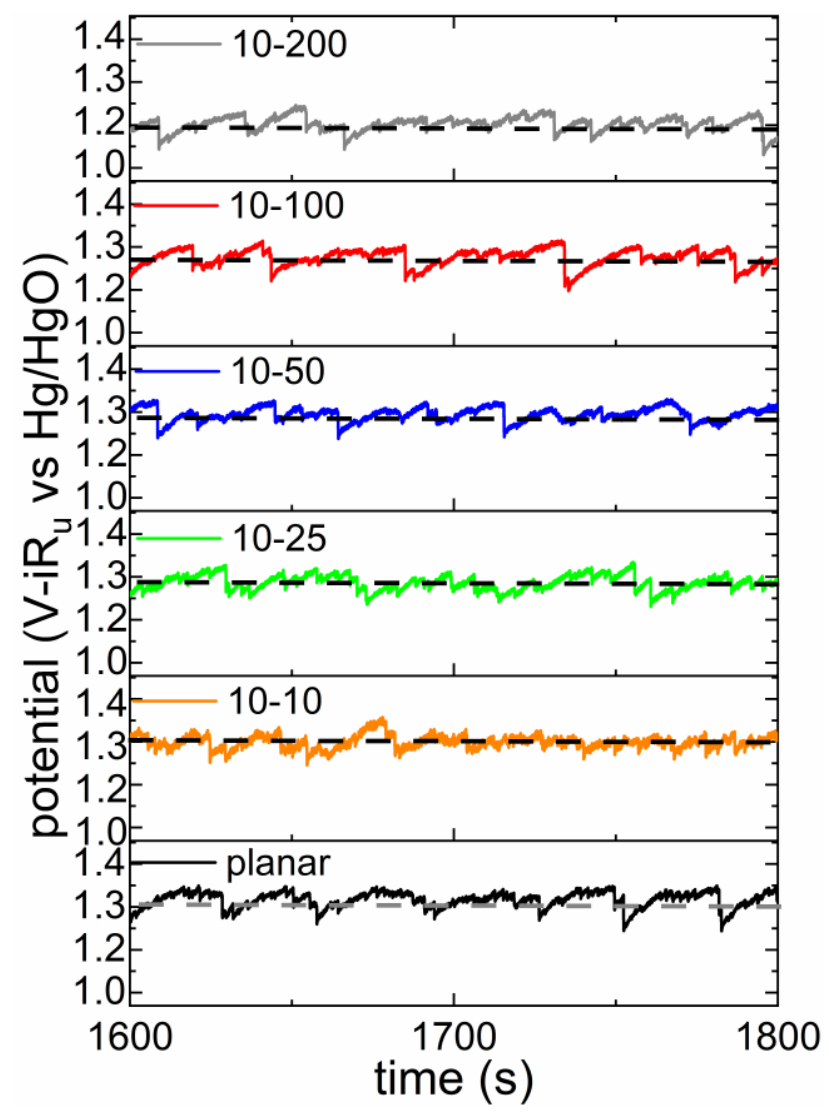

Figure S8. Representative CP plots acquired at $500 \mathrm{~mA} / \mathrm{cm}^{2}$ in purified $1 \mathrm{M} \mathrm{KOH}$. The type of electrode used in each measurement is labeled above the corresponding plot. These plots show a relatively short time-scale of $200 \mathrm{~s}$. 

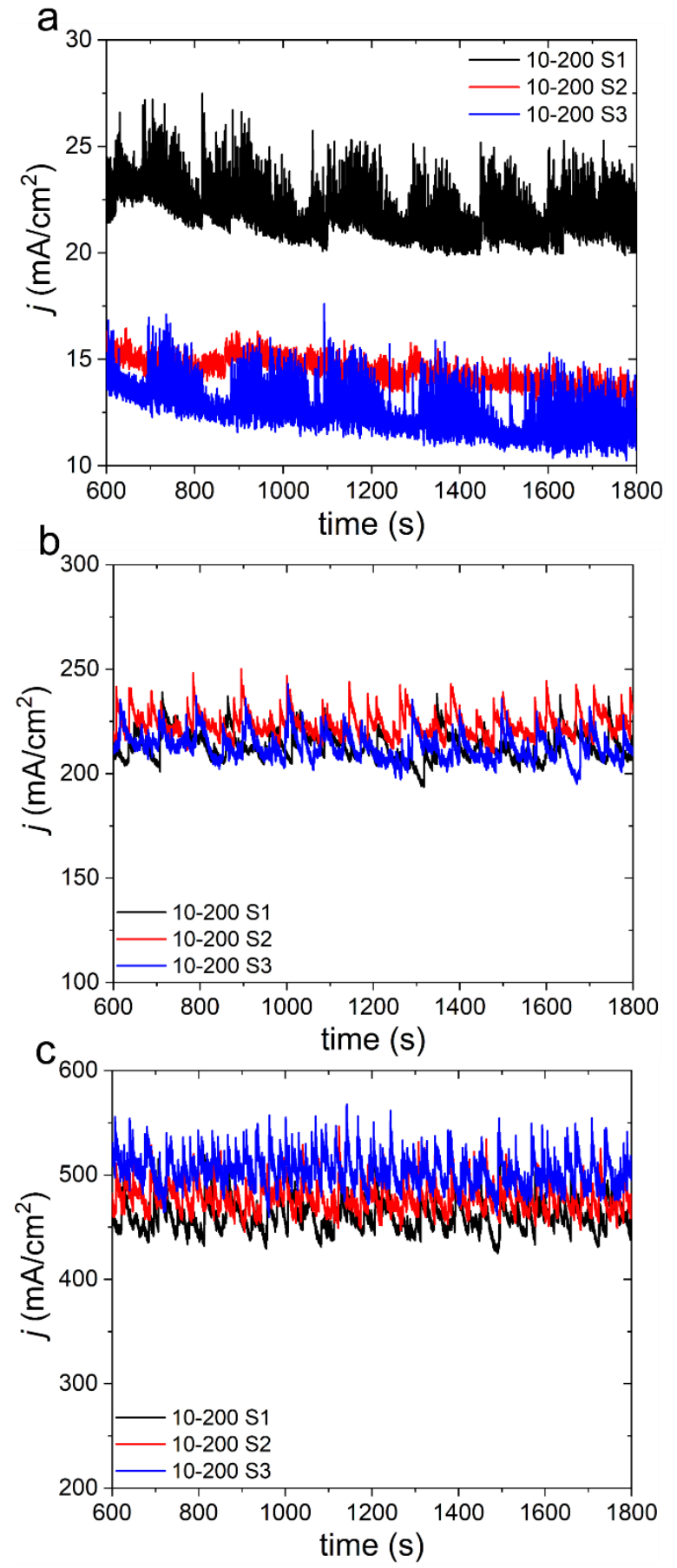

Figure S9. Chronoamperometry (CA) measurements acquired at (a) 1.0, (b) 1.4, and (c) $1.8 \mathrm{~V}$ vs $\mathrm{Hg} / \mathrm{HgO}$ for replicate samples each patterned with an array of 10-200 linear ridges. These plots depict the response of each sample from 600 to $1800 \mathrm{~s}$, following an initial period of equilibration for each experiment. 

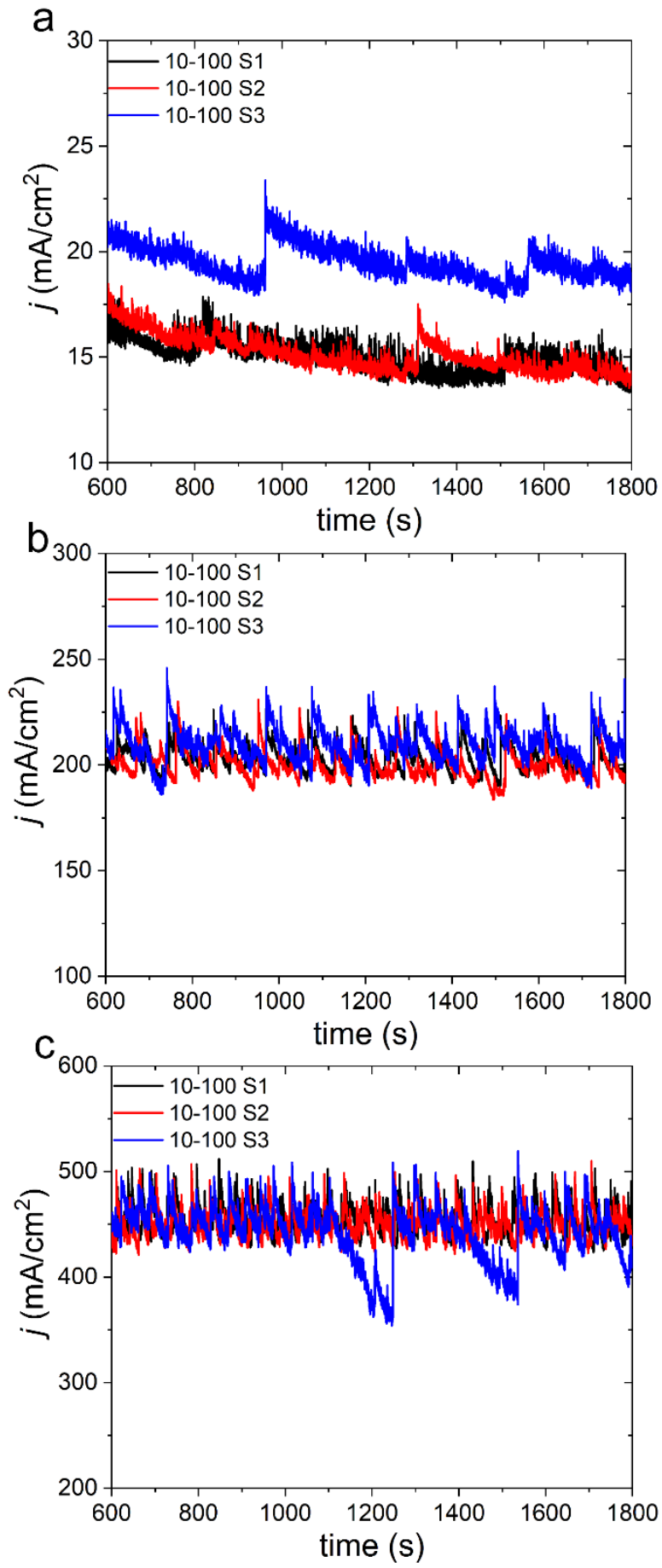

Figure S10. Chronoamperometry measurements acquired at (a) 1.0, (b) 1.4, and (c) $1.8 \mathrm{~V}$ vs $\mathrm{Hg} / \mathrm{HgO}$ for replicate samples each patterned with an array of 10-100 linear ridges. These plots depict the response of each sample from 600 to $1800 \mathrm{~s}$, following an initial period of equilibration for each experiment. 

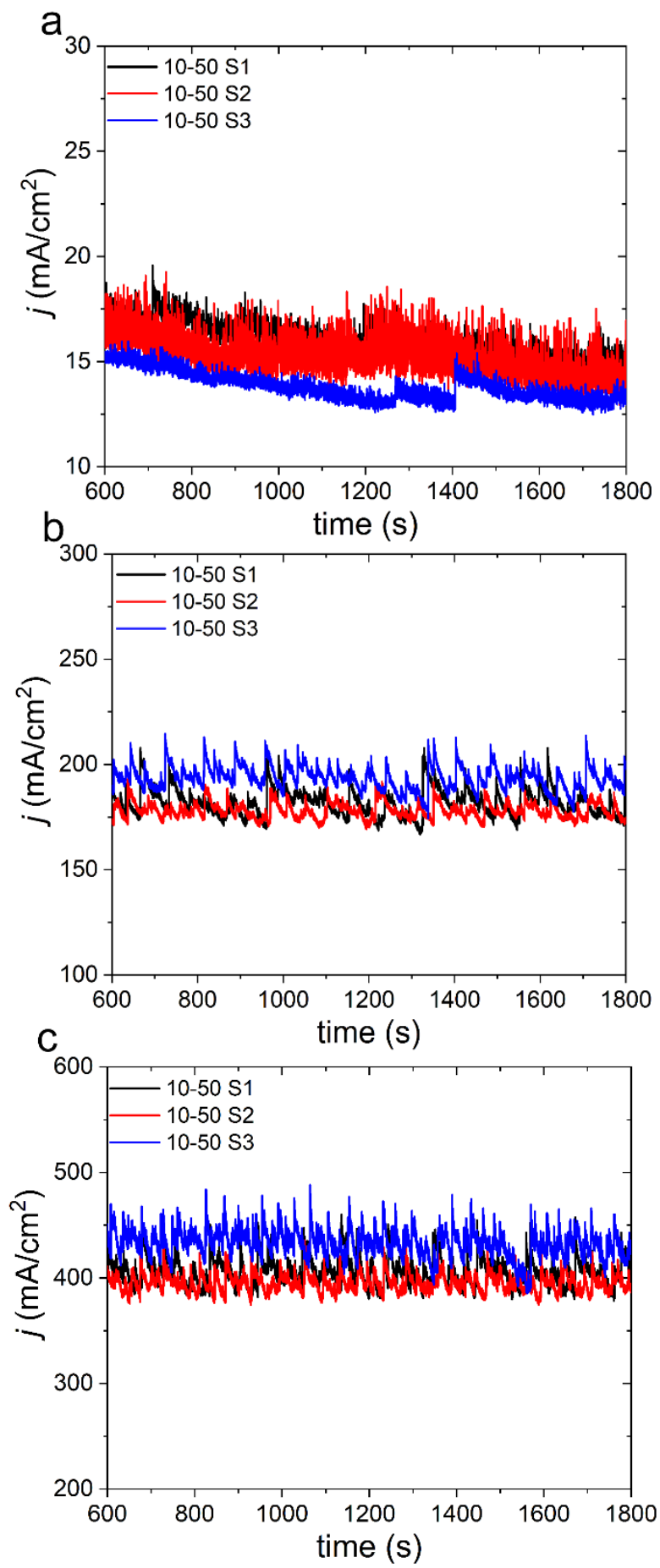

Figure S11. Plots of the CA measurements acquired at (a) 1.0, (b) 1.4 , and (c) $1.8 \mathrm{~V}$ vs $\mathrm{Hg} / \mathrm{HgO}$ for replicate samples each patterned with an array of 10-50 linear ridges. These plots depict the response of each sample from 600 to $1800 \mathrm{~s}$, following an initial period of equilibration for each experiment. 

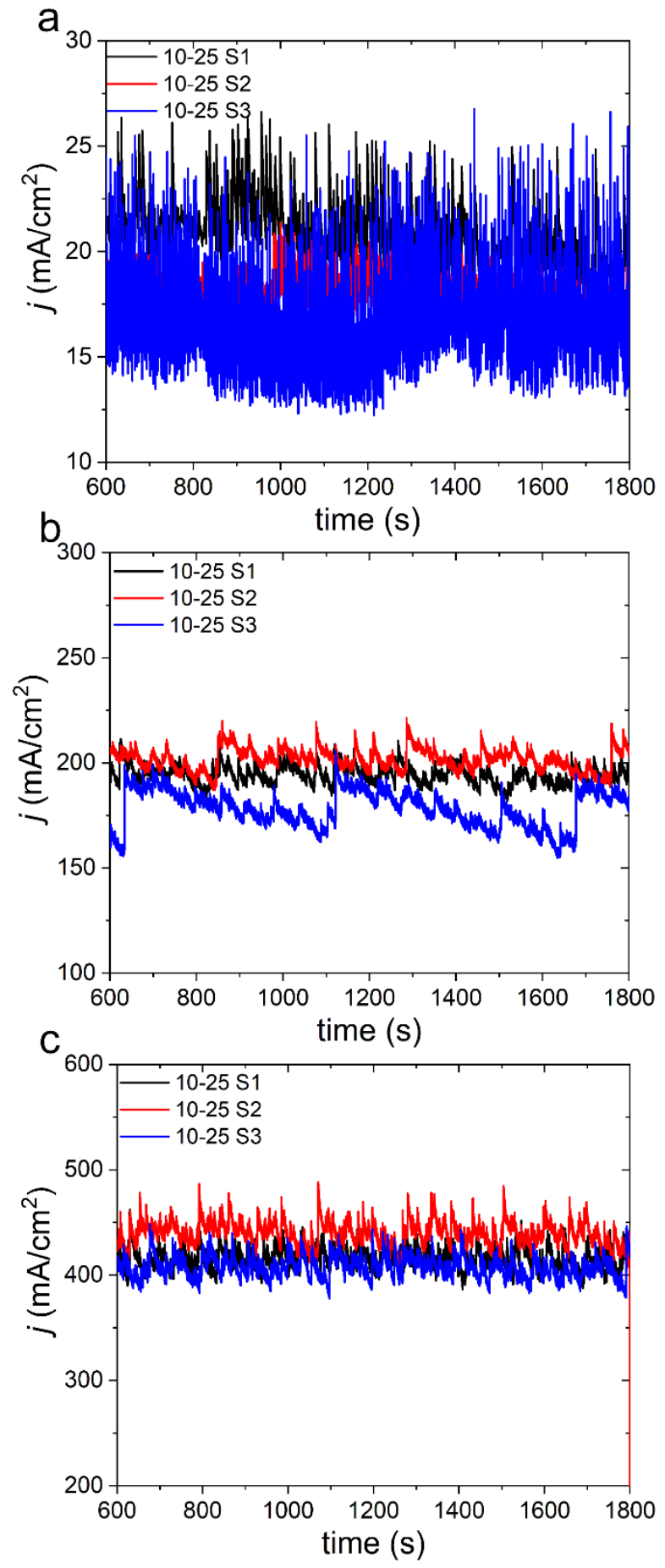

Figure S12. Plots of the CA measurements acquired at (a) 1.0 , (b) 1.4 , and (c) $1.8 \mathrm{~V}$ vs $\mathrm{Hg} / \mathrm{HgO}$ for replicate samples each patterned with an array of 10-25 linear ridges. These plots depict the response of each sample from 600 to $1800 \mathrm{~s}$, following an initial period of equilibration for each experiment. 

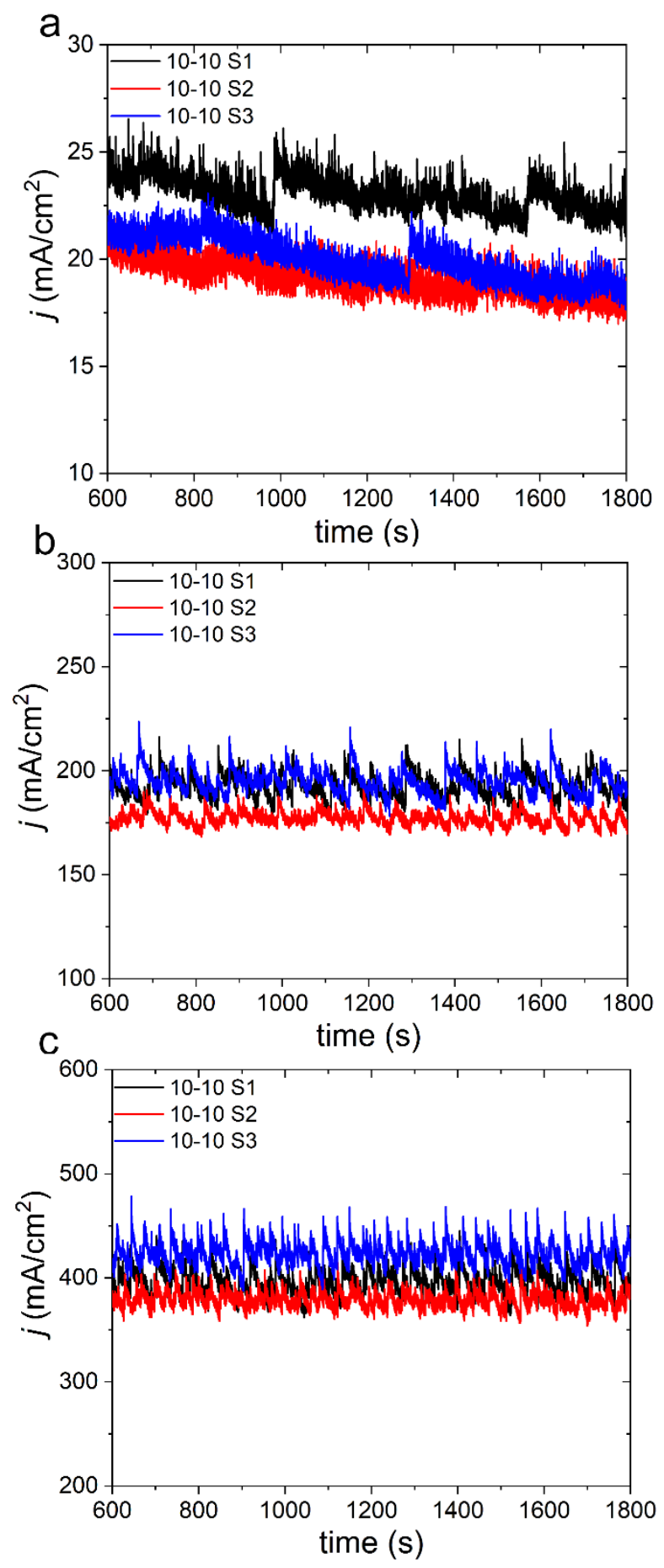

Figure S13. Plots of the CA measurements acquired at (a) 1.0 , (b) 1.4 , and (c) $1.8 \mathrm{~V} \mathrm{vs} \mathrm{Hg} / \mathrm{HgO}$ for replicate samples each patterned with an array of 10-10 linear ridges. These plots depict the response of each sample from 600 to $1800 \mathrm{~s}$, following an initial period of equilibration for each experiment. 

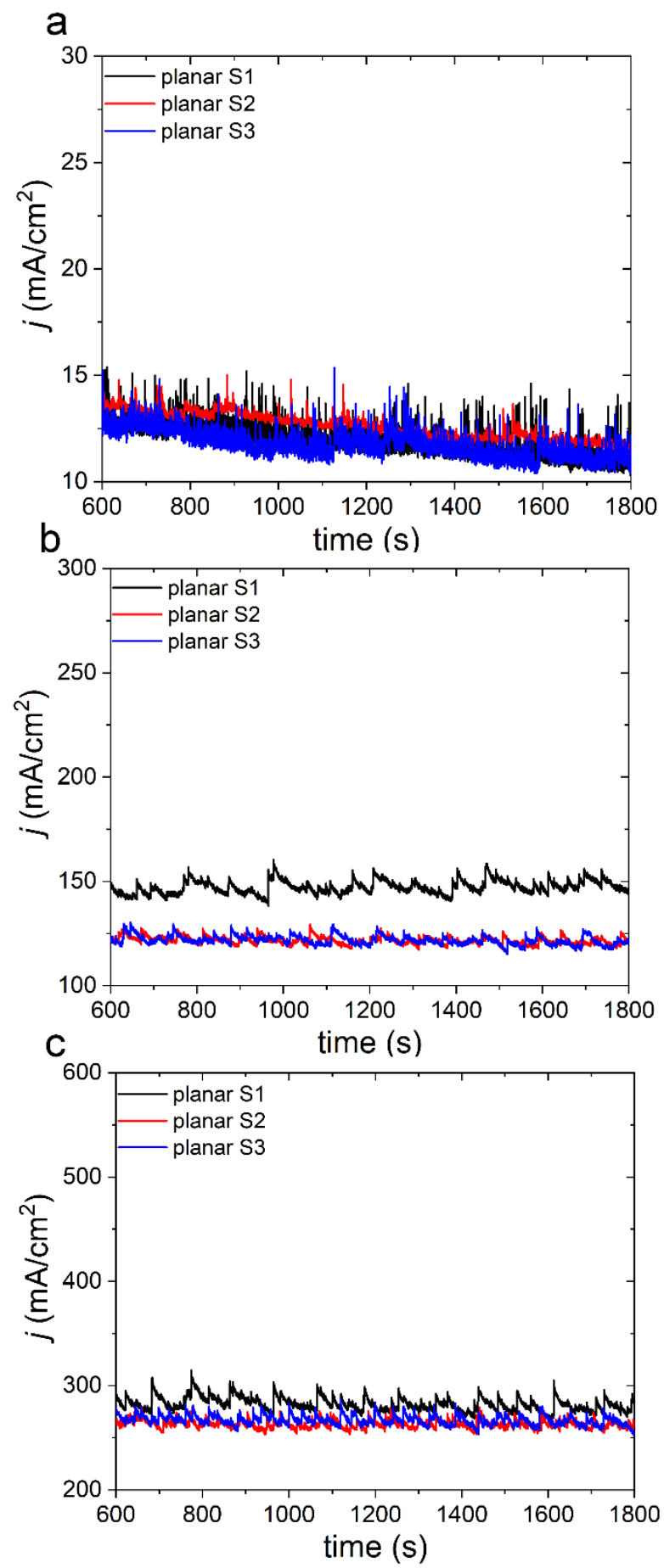

Figure S14. Plots of the CA measurements acquired at (a) 1.0 , (b) 1.4 , and (c) $1.8 \mathrm{~V} \mathrm{vs} \mathrm{Hg} / \mathrm{HgO}$ for the replicate samples with planar electrodeposited Ni surfaces. These plots depict the response of each sample from 600 to $1800 \mathrm{~s}$, following an initial period of equilibration for each experiment. 

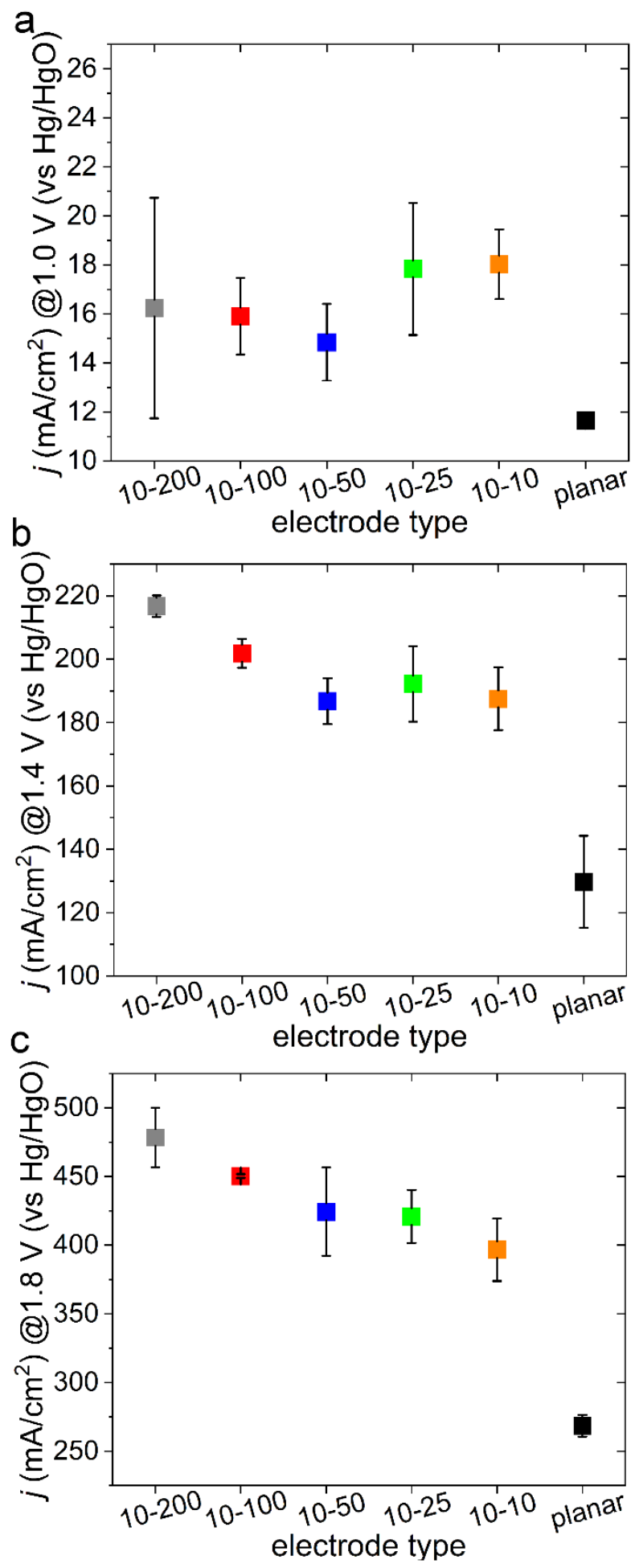

Figure S15. The mean current densities for the CA measurements acquired at (a) 1.0, (b) 1.4, and (c) $1.8 \mathrm{~V}$ (vs $\mathrm{Hg} / \mathrm{HgO}$ ) for all the replicate samples. The error bars are one standard deviation from the calculated mean values. 

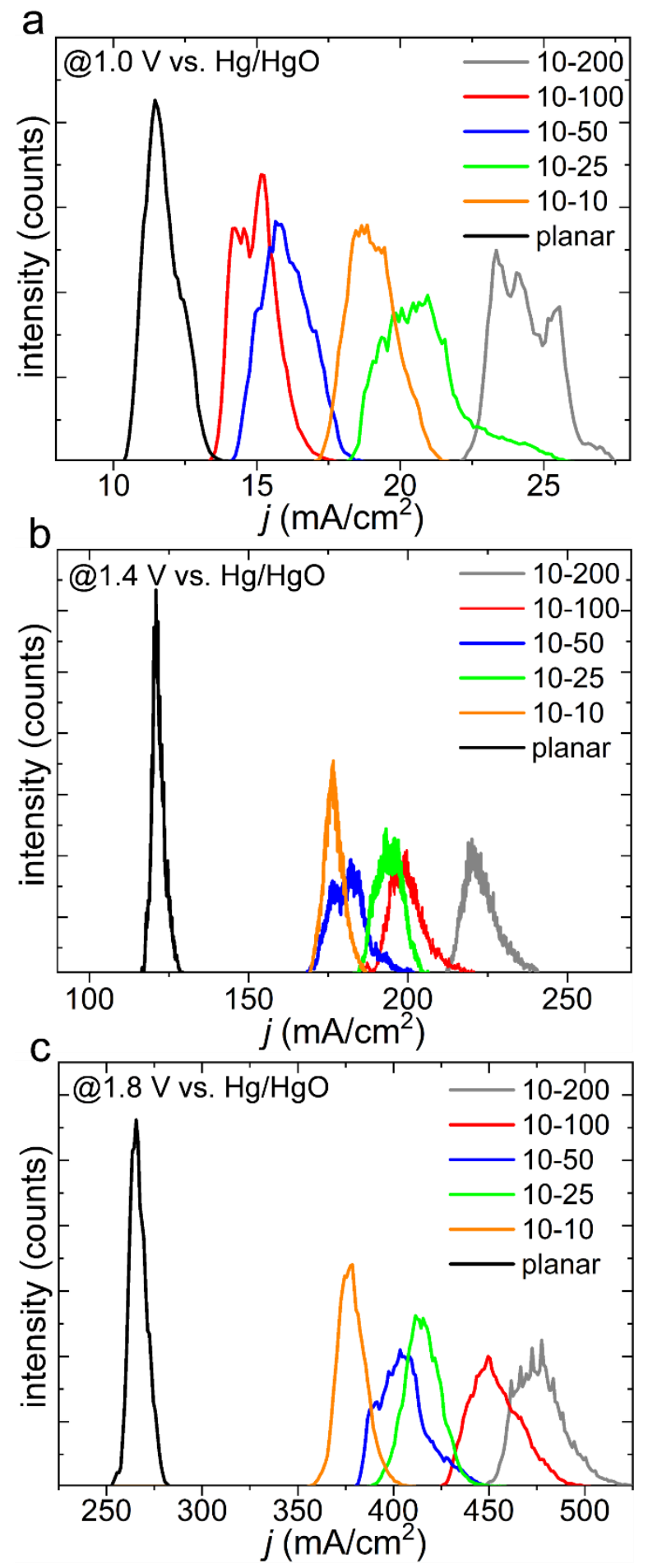

Figure S16. Histograms prepared from the CA measurements acquired at (a) 1.0, (b) 1.4, and (c) $1.8 \mathrm{~V}(\mathrm{vs} \mathrm{Hg} / \mathrm{HgO})$ for the planar electrodeposited Ni surfaces and each type of sample patterned with arrays of linear ridges as noted in the legends. 


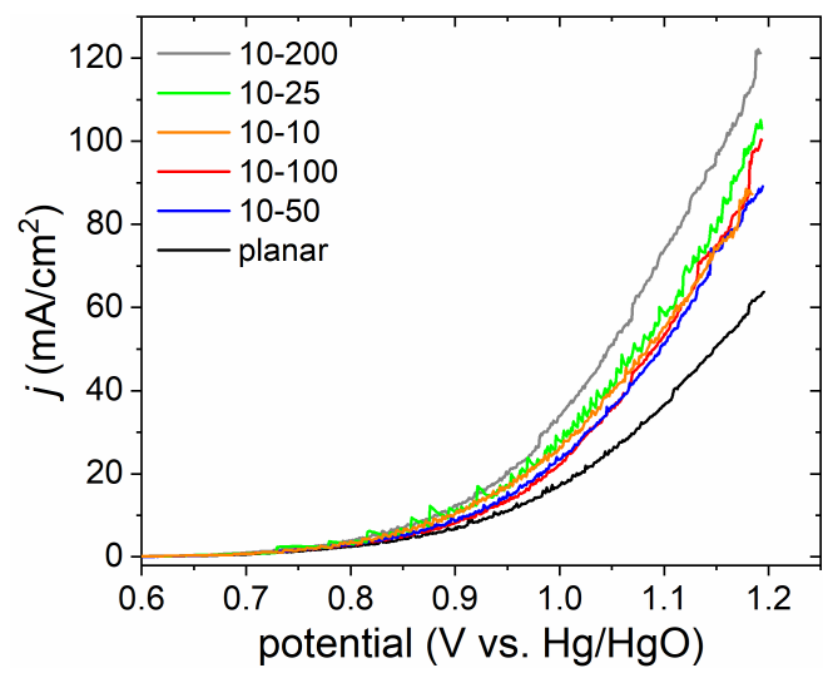

Figure S17. The performance of the electrodes patterned with arrays of parallel microscale features towards the oxygen evolution reaction (OER) assessed by linear sweep voltammetry (LSV). The response curve for a planar electrode of $\mathrm{Ni}$ is included for comparison. Measurements were acquired in purified $1 \mathrm{M} \mathrm{KOH}$ at a scan rate of $1 \mathrm{mV} / \mathrm{s}$. The current is normalized for each sample to the theoretical electrochemically active surface area ( $\left.A_{\text {theortical }}\right)$ according to the values reported in Table 1. 

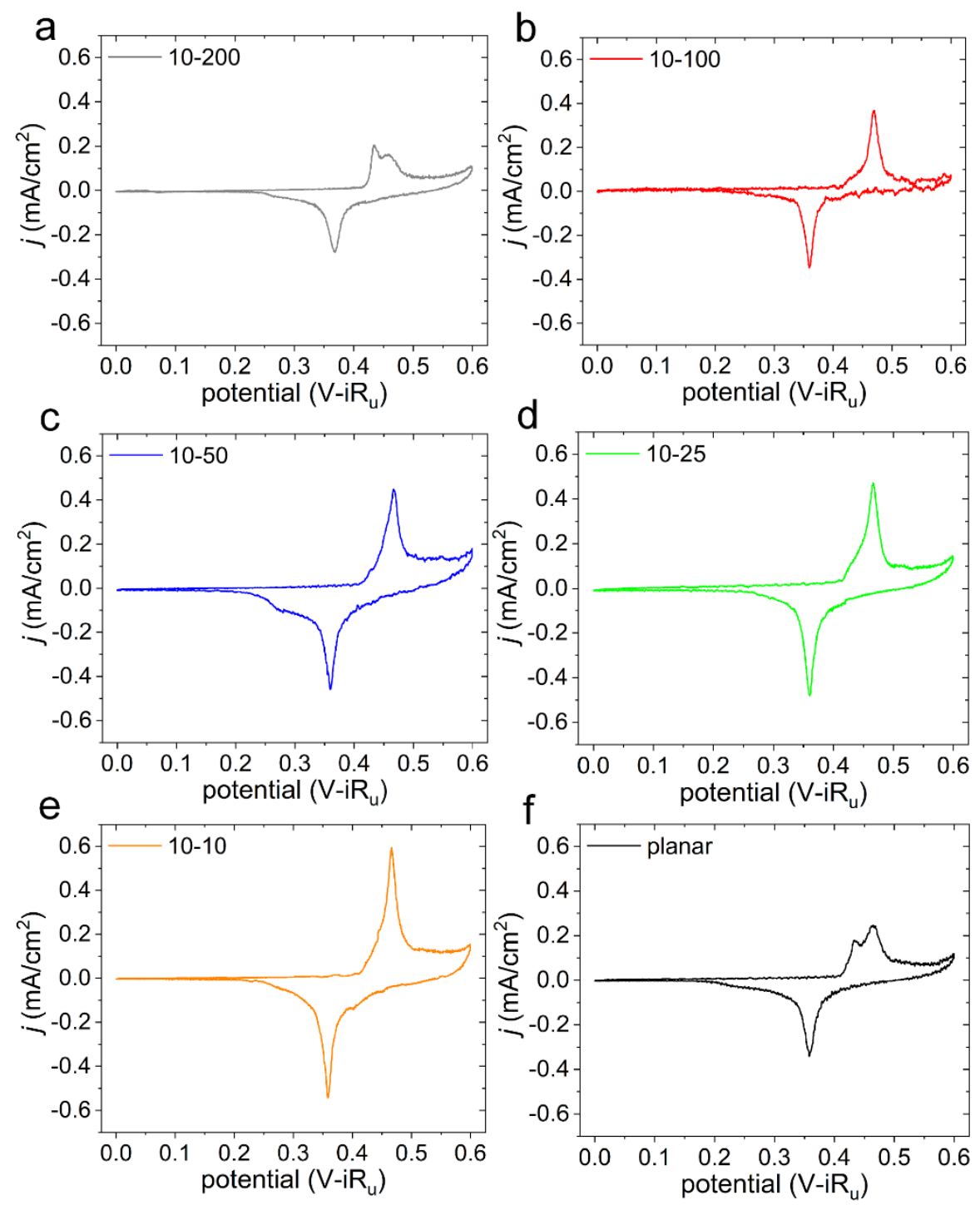

Figure S18. Cyclic voltammetry scans acquired directly after electrochemical testing for each type of Ni electrode: (a) 10-200; (b) 10-100; (c) 10-50; (d) 10-25; (e) 10-10; and (f) planar electrodes. The $\mathrm{CV}$ measurements were acquired at a scan rate of $1 \mathrm{mV} / \mathrm{s}$ in a purified solution of $1 \mathrm{M} \mathrm{KOH}$. The measured currents were normalized to $A_{\text {theoretical. }}$. 
Table S1. A comparison of the electrochemically active surface area $\left(A_{\text {ecsa }}\right)$ values calculated for each type of electrode.

\begin{tabular}{cc}
\hline electrode type $*$ & $A_{\text {ecsa }}\left(\mathrm{cm}^{2}\right)^{\varphi}$ \\
\hline \hline $10-200$ & $14 \pm 2$ \\
$10-100$ & $13 \pm 3$ \\
$10-50$ & $18 \pm 7$ \\
$10-25$ & $20 \pm 3$ \\
$10-10$ & $26 \pm 2$ \\
planar & $15 \pm 1$
\end{tabular}

* The description of the electrode type refers to the target width of the ridges and the distance between each ridge, respectively.

${ }^{\varphi}$ The $A_{\text {ecsa }}$ were derived using the charge density method to analyze the cathodic reduction peaks in the CV scans. ${ }^{1-4}$ The mean $A_{\text {ecsa }}$ were obtained by integrating the cathodic peaks to determine the quantities of charge passed $\left(Q_{\text {cat }}\right)$ from the $C V$ plots acquired at $1 \mathrm{mV} / \mathrm{s}$. The error represents one standard deviation from the calculated mean values. 

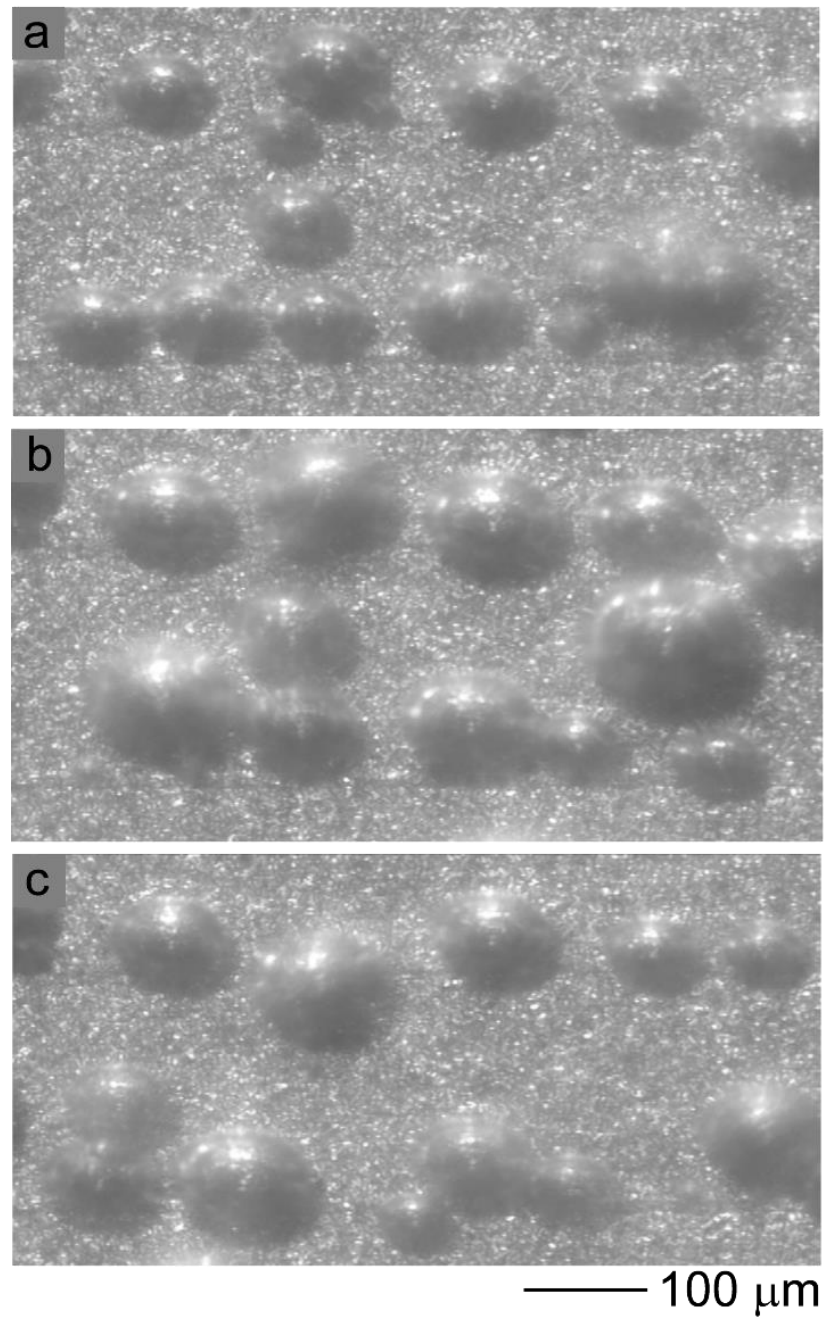

Figure S19. Representative frames from the video footage acquired for the 10-200 type electrode. Each frame in $(\mathrm{a}-\mathrm{c})$ was acquired prior to the onset of bubble release from the electrode. 

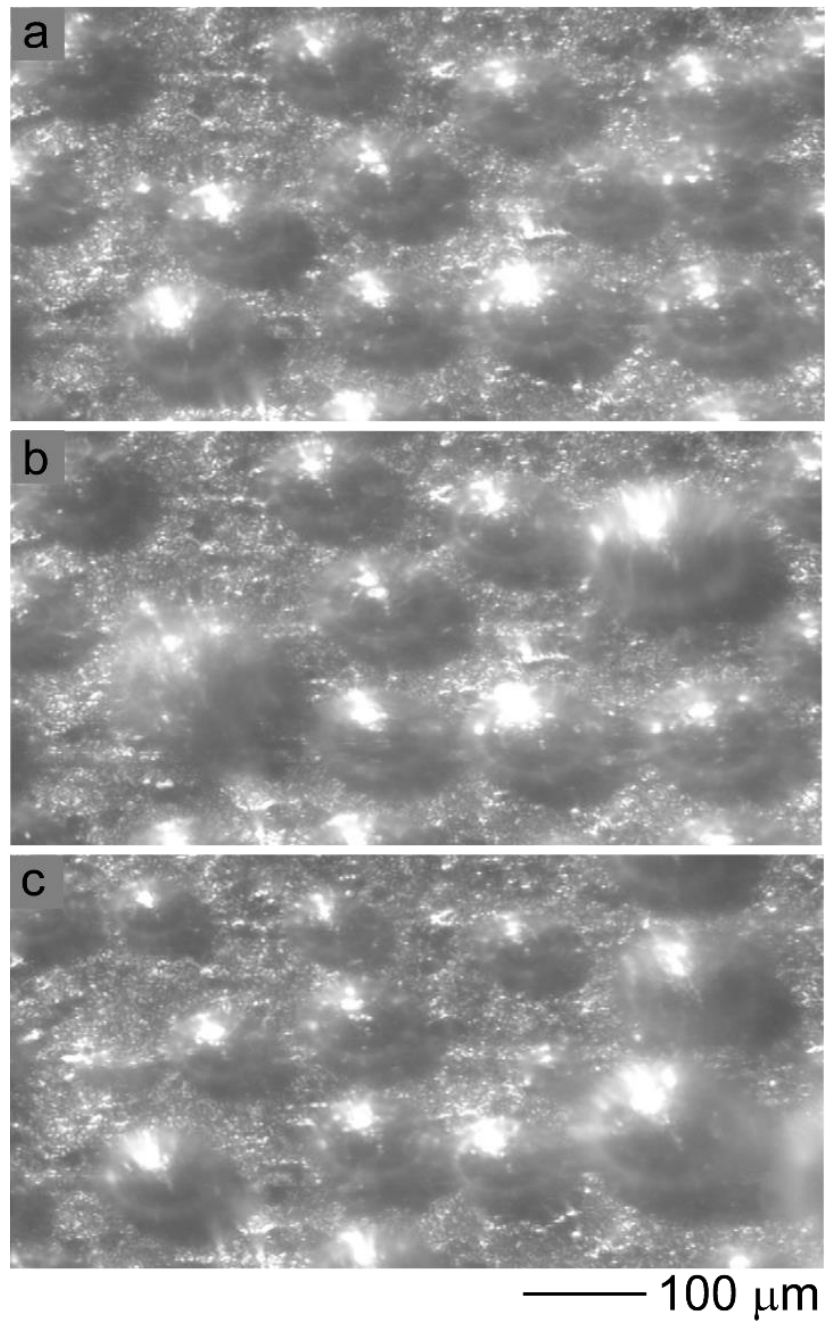

Figure S20. Representative frames from the video footage acquired for the 10-100 electrode. Each frame in (a-c) was acquired prior to the onset of bubble release from the electrode. 

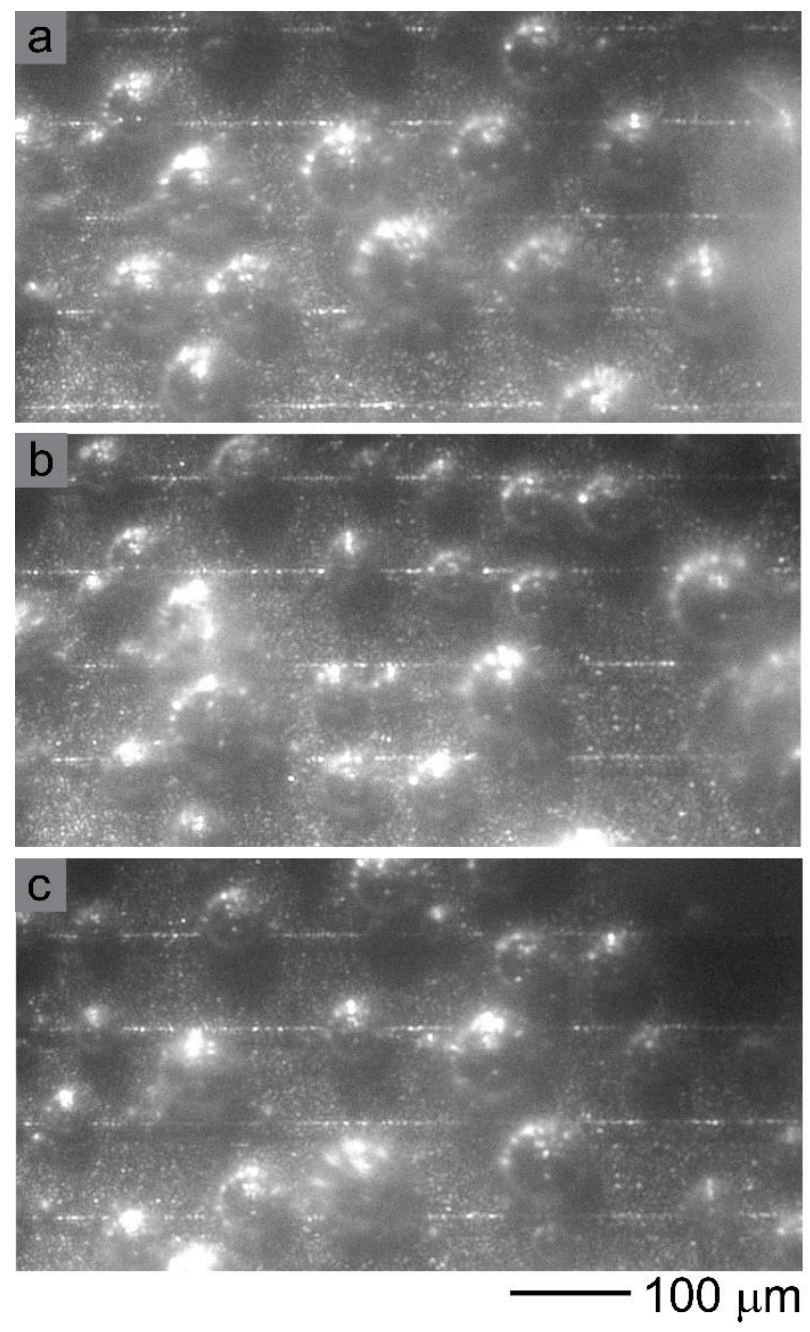

Figure S21. Representative frames from the video footage acquired for the 10-50 electrode. Each frame in (a-c) was acquired prior to the onset of bubble release from the electrode. 

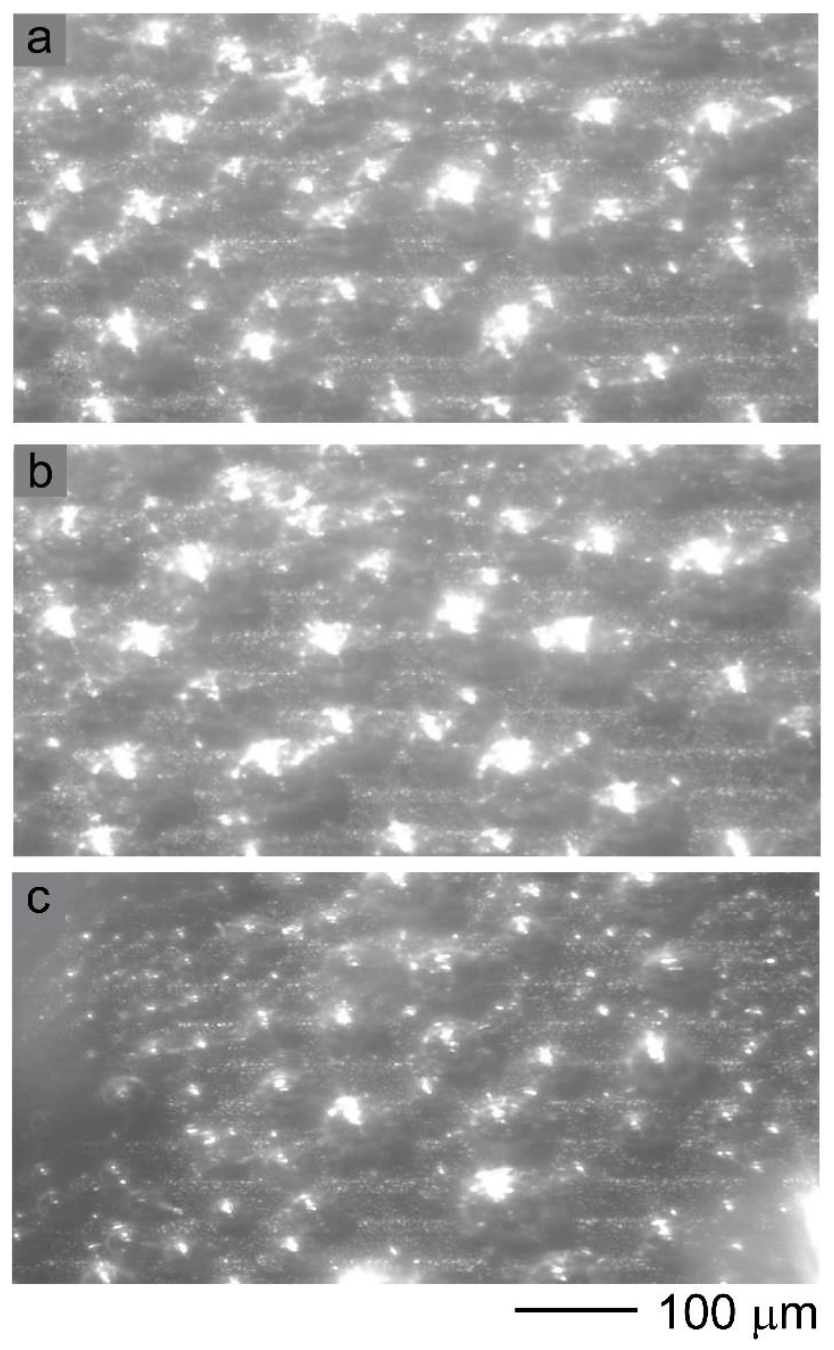

Figure S22. Representative frames from the video footage acquired for the 10-25 electrode. Each frame in (a-c) was acquired prior to the onset of bubble release from the electrode. 

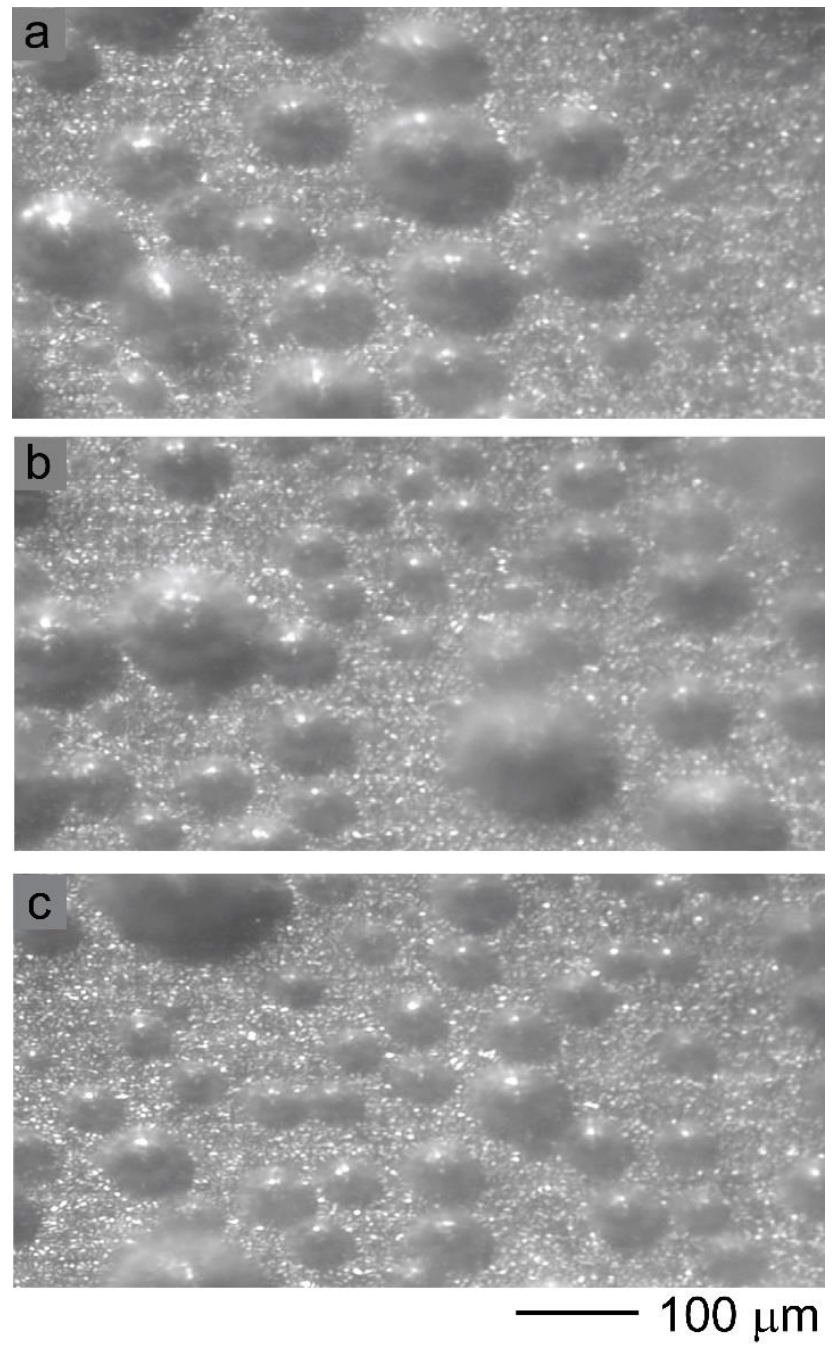

Figure S23. Representative frames from the video footage acquired for the 10-10 electrode. Each frame in (a-c) was acquired prior to the onset of bubble release from the electrode. 

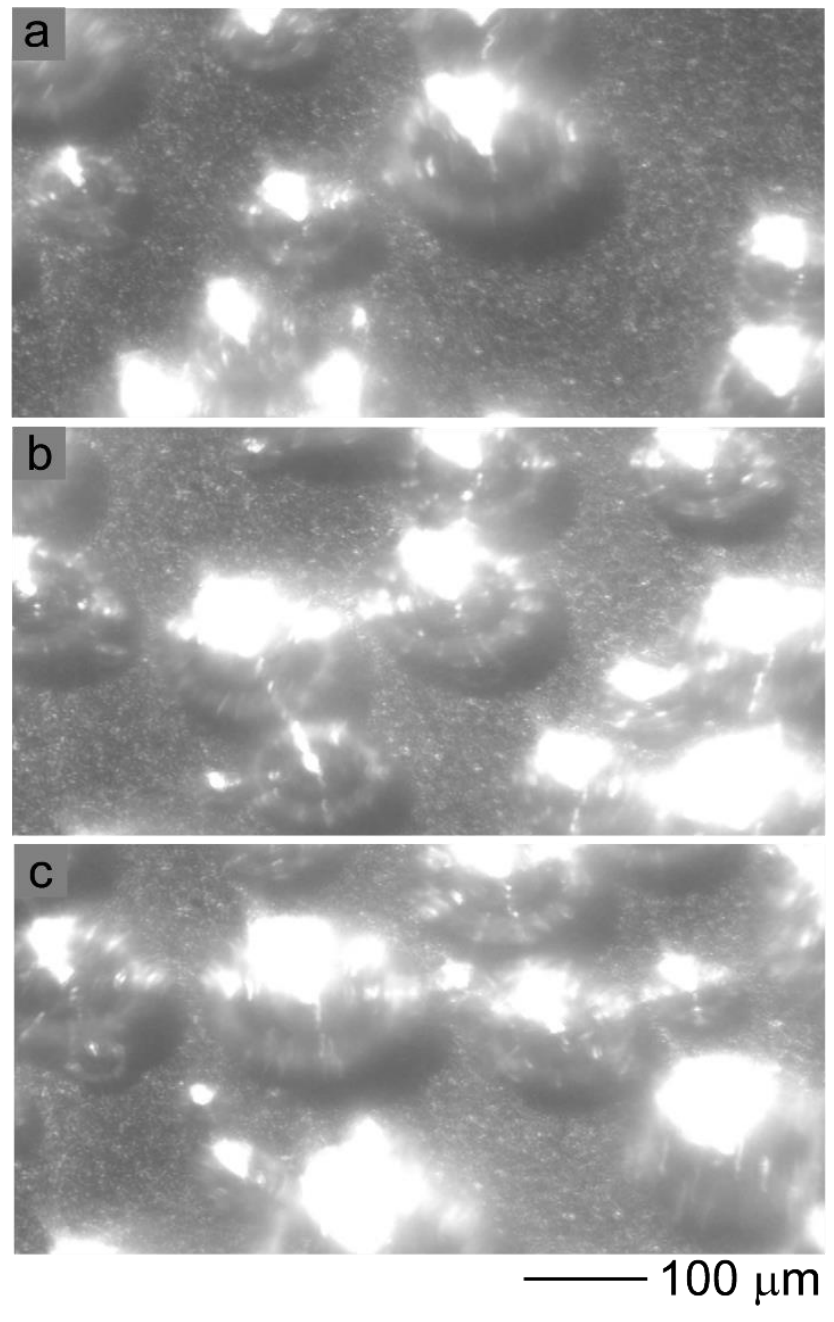

Figure S24. Representative frames from the video footage acquired for the planar electrode. Each frame in (a-c) was acquired prior to the onset of bubble release from the electrode. 


\section{Section S2.}

The fractional bubble coverage, $\Theta$, was determined by measuring the area occupied by the bubbles, $B_{A}$, directly prior to their departure from the electrode surface. The geometric surface area, $A$, was divided by the value acquired for $B_{A}$ in Equation $\mathrm{S} 1$. The periodic release of multiple bubbles from the electrodes patterned with arrays of linear ridges enabled a relatively accurate determination of the bubble coverage in comparison to the planar electrode, which exhibited relatively non-uniform events for the release of bubbles from their surfaces.

$$
\Theta=\frac{B_{A}}{A}
$$

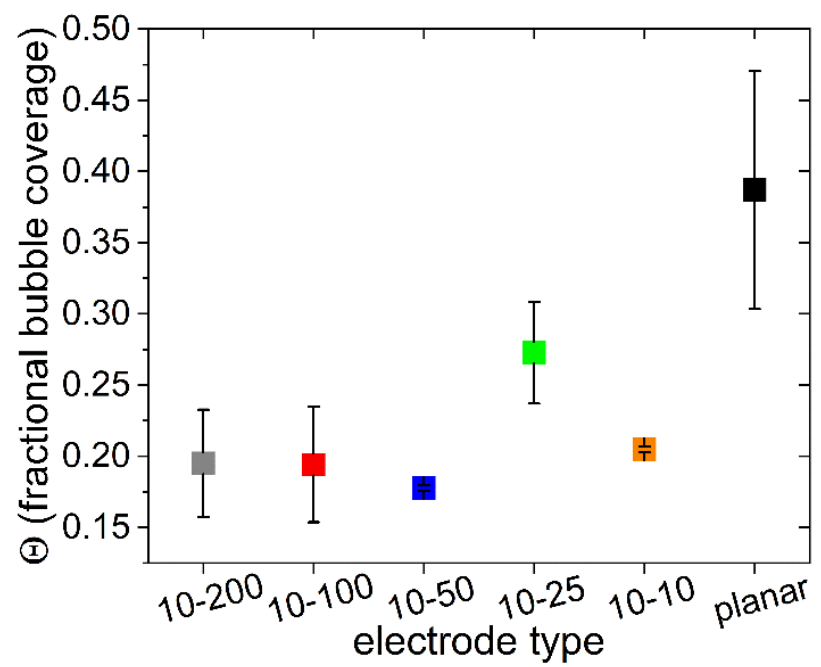

Figure S25. The fractional bubble coverage of each type of electrode as determined using still frames from the movies of bubble evolution that were acquired at $1.8 \mathrm{~V}(\mathrm{vs} \mathrm{Hg} / \mathrm{HgO})$. The error bars represent one standard deviation from the mean values calculated using Equation S1. The error bars for the 10-50 and 10-10 electrode are proportional to the symbol used in the plot. 


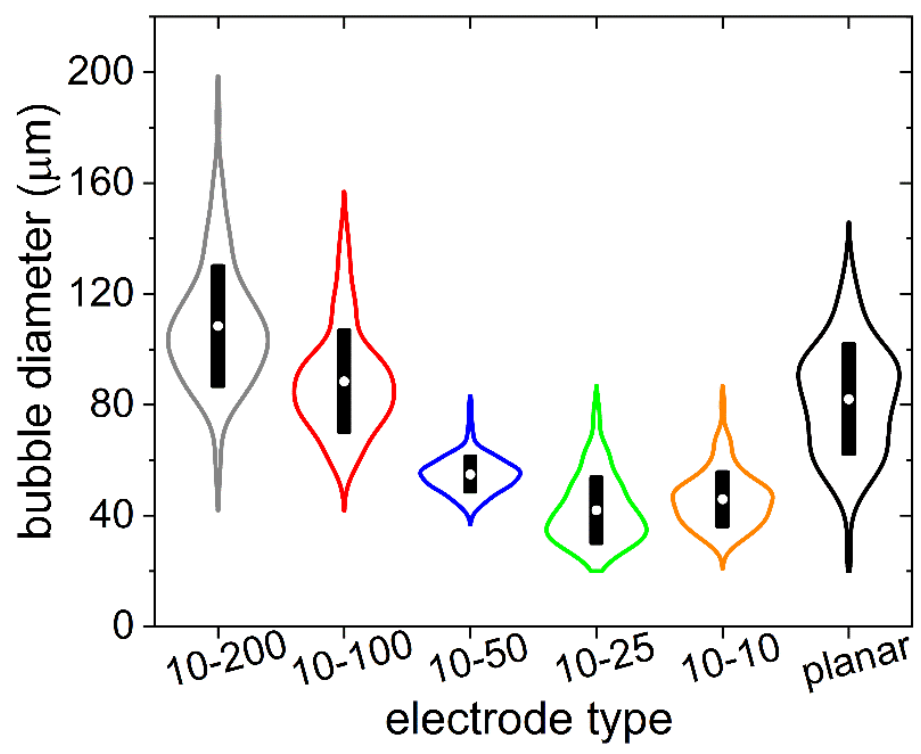

Figure S26. The mean departure diameters of bubbles were measured from still frames acquired directly prior to release events from the electrode surfaces. The mean values depicted by the white circles were calculated from at least 100 individual bubble measurements for each type of electrode. The violin plot shows the probability distribution of the bubble diameters for each data set. The rectangular errors bars represent one standard deviation from the calculated mean values. 
The bubble residence time, $t_{r}$, was calculated from the high speed video data acquired at $1.8 \mathrm{~V}$ (vs $\mathrm{Hg} / \mathrm{HgO}$ ) for each type of electrode. ${ }^{5,6}$ The gas evolution efficiency, $f_{g}$, was calculated using the values for the fractional bubble coverage (Figure S25), while the Faradaic efficiency, $\Phi_{B}$, used in these calculations was obtained from the literature for a pure Ni electrode. ${ }^{7}$ The vapor partial pressure for the electrolyte, $P_{s}$, was also obtained from the literature. ${ }^{8}$ The average bubble radius, $R_{r}$, directly prior to detachment, the current, $I$, the electrode area, $A$, the ideal gas constant, $R_{m}$, the temperature, $T$, Faraday's constant, $F$, the bubble volume prior to detachment, $V_{r}$, the stoichiometric ratio of the number of electrons to the number of $\mathrm{O}_{2}$ molecules, $v_{\mathrm{e}} / v_{B}$, and the atmospheric pressure, $P$, were included in the following equation:

$$
t_{r}=\frac{\Theta R_{r}}{\frac{\pi R_{r}^{3}}{2 V_{r}} f_{g} \Phi_{B} \frac{I /{ }_{A} R_{m} T}{v_{e} / v_{B} F\left(P-P_{S}\right)}}
$$

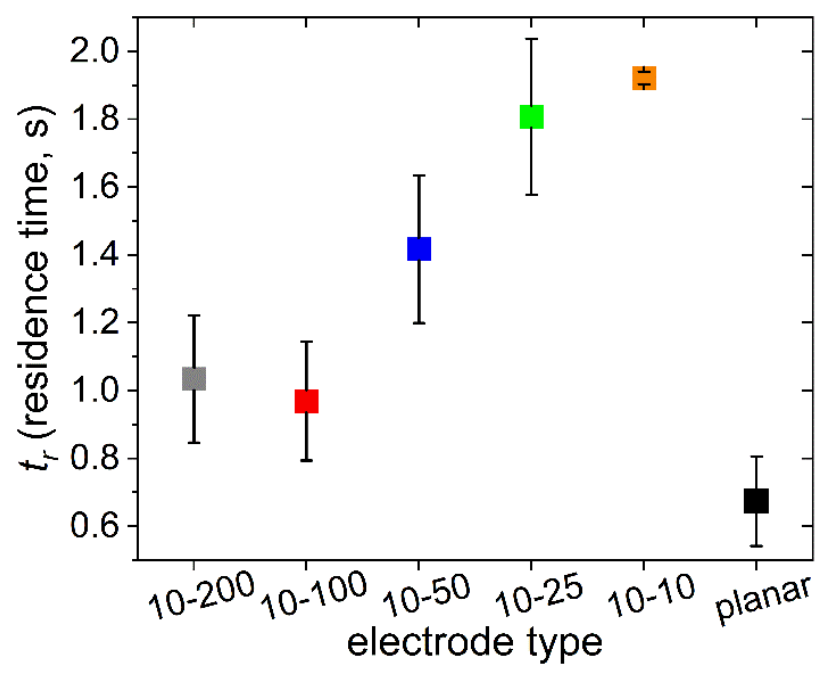

Figure S27. Mean residence times of the bubbles on each type of electrode as calculated using Equation S2. The error bars represent one standard deviation from the mean values. The error bars for the 10-10 electrode are proportional to the symbol used in the plot. 
The volumetric flux, $V_{g}$, of the gaseous product was determined from the high speed camera data acquired at $1.8 \mathrm{~V}$ (vs $\mathrm{Hg} / \mathrm{HgO}$ ). The average number of adhered bubbles, $n_{r}$, the bubble volume prior to detachment, $V_{r}$, and the residence time, $t_{r}$, were included in the following equation: ${ }^{6}$

$$
V_{g}=\frac{n_{r} V_{r}}{t_{r}}
$$


The theoretical activation overpotential, $\eta_{\mathrm{a}}$, can be described in terms of the average fractional bubble coverage, $\Theta$, Faraday's constant, $F$, the ideal gas constant, $R_{m}$, and the temperature, $T$, according to the following equation: ${ }^{5}$

$$
\eta_{\mathrm{a}}=\frac{R_{m} T}{F} \ln (1-\Theta)
$$

(Equation S4)

The theoretical ohmic overpotential, $\eta_{\mathrm{ohm}}$, can be described by the mass transfer resistances associated with both the bubbles on the electrode surfaces and the freely moving bubbles in the electrolyte. ${ }^{9,10}$ The presence of bubbles imposes a modification to the conductivity of the electrolyte, $\kappa$. The thickness of the bubble layer, $T_{b}$, and the volume fraction of gas, $V_{b}$, were estimated from the average bubble diameter measurements acquired during the high speed camera analyses. The average current over the duration of the experiment, $I$, the area of the electrode, $A$, the interelectrode distance, $d_{e}$, and the average volume fraction of gas, $\Theta$, were used in the following equation:

$$
\eta_{\mathrm{ohm}}=\frac{I d_{e}}{A \kappa}\left[\frac{T_{b}}{d_{e}}\left(1-\frac{\Theta}{1.5}\right)^{-1.5}+\left(1-\frac{T_{b}}{d_{e}}\right)\left(1-V_{b}\right)^{-1.5}\right]
$$

The theoretical concentration overpotential, $\eta_{\text {conc }}$, addresses the concentration gradients induced by the presence of bubbles. ${ }^{11}$ The degree of supersaturation in the electrolyte prior to bubble nucleation at the electrode surface, $C_{g}$, was estimated based on prior studies relative to the concentration of gas at $1 \mathrm{~atm}$ pressure, $C_{\text {sat }}{ }^{12}$ The ideal gas constant, $R_{m}$, the temperature, $T$, the number of electrons, $n$, and Faraday's constant, $F$, were used in the expression for the $\eta_{\text {conc. }}$ This overpotential was found to be approximately $36 \mathrm{mV}$ for each experiment.

$$
\eta_{\text {conc }}=\frac{R_{m} T}{n F} \ln \frac{C_{g}}{C_{\text {sat }}}
$$


The potential difference (or voltage difference) from the thermodynamically determined potential and the realized potential $(\Delta \mathrm{V})$ can be described in part by contributions from each of the theoretical, bubble-related overpotentials:

$$
\Delta V=\eta_{\mathrm{a}}+\eta_{\mathrm{ohm}}+\eta_{\text {conc }}
$$

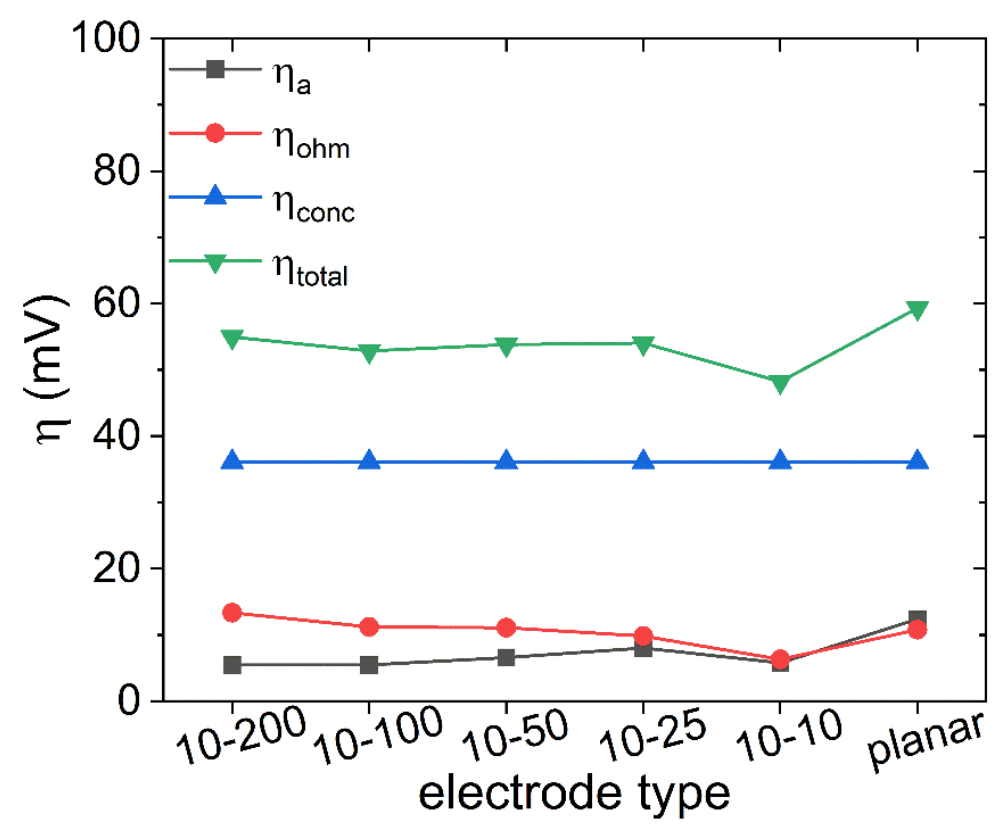

Figure S28. The theoretical overpotentials calculated for both the activation $\left(\mathrm{\eta}_{\mathrm{a}}\right)$, ohmic $\left(\mathrm{\eta}_{\mathrm{ohm}}\right)$, and the concentration ( $\eta_{\text {conc }}$ ) are plotted, as well as the resulting summation of each contribution to the overpotential ( $\eta_{\text {total }}$ ), as a function of the type of electrodes analyzed in this study.

In general, the theoretical overpotentials are similar for each of the electrodes but are the lowest for the 10-10 type electrodes and the highest for the planar electrodes. The trends predicted by the theoretical overpotentials are, however, distinct from the trends observed in the measured results. The lower overpotentials predicted for the 10-10 type electrodes are not capturing the influences 
of trapped gas bubbles and the presence of smaller bubbles at the microscale ridges. More regular and synchronous bubble release from the 10-200 electrodes likely had a significant improvement on the mixing of the electrolyte near the surfaces of these electrodes and an increase in their volumetric gas evolution. It is also likely that there are differences in concentration gradients that could exist between the different types of electrodes as a result of these factors. 


\section{List of Symbols for Sections S2:}

A, geometric electrode area $\left(\mathrm{m}^{2}\right)$

$B_{A}, \quad$ area occupied by a bubble $\left(\mathrm{m}^{2}\right)$

$d_{e}, \quad$ interelectrode distance (m)

$F, \quad$ Faraday constant, $F=96485 \mathrm{~A} \mathrm{~s} \mathrm{~mol}^{-1}$

$f_{g}, \quad$ gas evolution efficiency

$I, \quad$ current (A)

$n_{r}, \quad$ number of adhered bubbles

$P, \quad$ pressure $\left(\mathrm{kg} \mathrm{m}^{-1} \mathrm{~s}^{-2}\right)$

$P_{s}, \quad$ vapor pressure of solvent $\left(\mathrm{kg} \mathrm{m}^{-1} \mathrm{~s}^{-2}\right)$

$R_{m}, \quad$ ideal gas constant, $R_{m}=8.314 \mathrm{~kg} \mathrm{~m}^{2} \mathrm{~s}^{-2} \mathrm{~mol}^{-1} \mathrm{~K}^{-1}$

$R_{r}, \quad$ average bubble radius directly prior to detachment from the surfaces (m)

$T, \quad$ temperature $(\mathrm{K})$

$T_{b}, \quad$ thickness of the bubble layer on the electrode (m)

$t_{r}, \quad$ residence time (s)

$V_{g}, \quad$ volumetric flux $\left(\mathrm{m}^{3} \mathrm{~s}^{-1}\right)$

$V_{r}, \quad$ bubble volume directly prior to detachment from the surfaces $\left(\mathrm{m}^{3}\right)$

$V_{b}, \quad$ volume fraction of gas in the electrolyte

$v_{e} / v_{B}$, stoichiometric ratio of the number of electrons to the number of $\mathrm{O}_{2}$ molecules produced

$\Phi_{B}, \quad$ Faradaic efficiency

$\Theta$, fractional bubble coverage

$\eta_{\mathrm{a}}, \quad$ activation overpotential

$\eta_{\text {ohm }}$, ohmic overpotential

$\eta_{\text {conc, }}$ concentration overpotential

$\kappa, \quad$ conductivity of the electrolyte $\left(\mathrm{A} \mathrm{V}^{-1} \mathrm{~m}^{-1}\right)$

$C_{g}, \quad$ interfacial supersaturation level at the electrode surface

$C_{\text {sat }}$, relative concentration of dissolved gas in the bulk electrolyte at $1 \mathrm{~atm}$ 


\section{Section S3.}
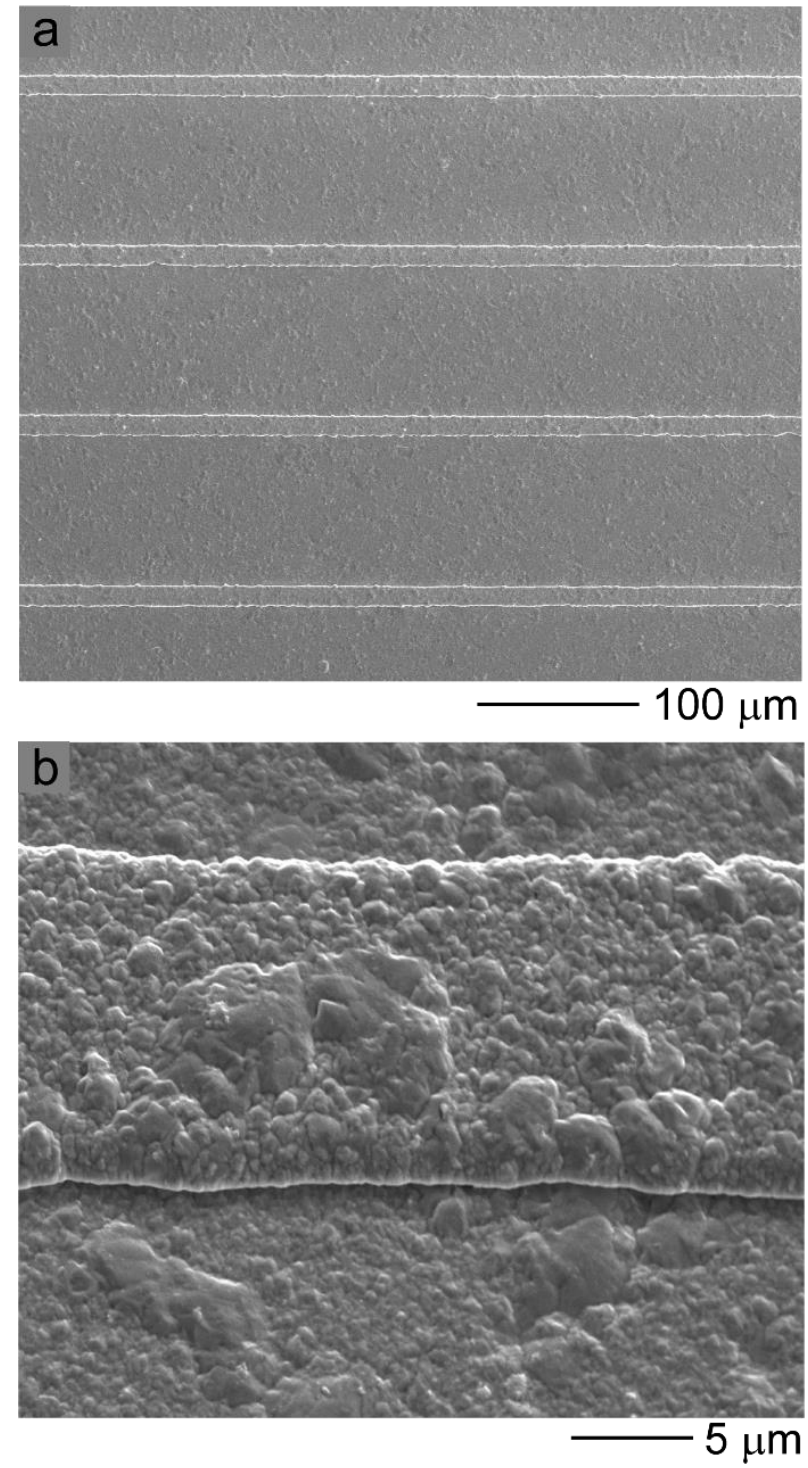

Figure S29. Representative SEM images of the 10-100 electrode acquired after the electrochemical measurements. The image in (a) was captured at a low magnification while the image for (b) was acquired at a $45^{\circ}$ tilt and at a higher magnification. 


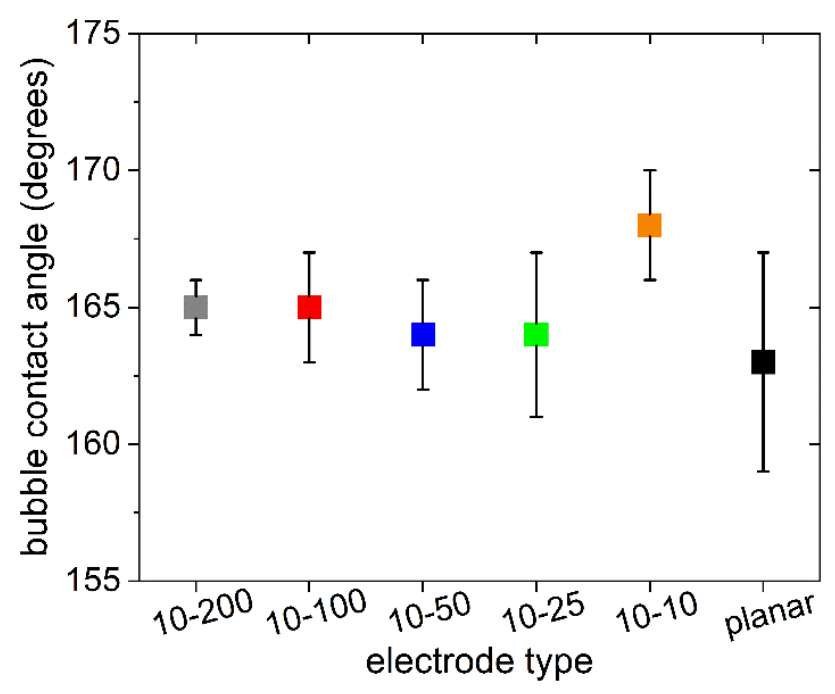

Figure S30. The mean gas bubble contact angles (BCA) calculated using six separate measurements for each type of electrode. The error bars represent one standard deviation from the calculated mean values. The measurements were acquired after electrochemical testing. 
Table S2. Gas Bubble Contact Angle Measurements for the Microscale, Linear Ridges

\begin{tabular}{cc} 
electrode type $*$ & mean gas bubble contact angle $(\mathrm{BCA})^{\ddagger}$ \\
\hline \hline $10-200$ & $165^{\circ} \pm 1^{\circ}$ \\
$10-100$ & $165^{\circ} \pm 2^{\circ}$ \\
$10-50$ & $164^{\circ} \pm 2^{\circ}$ \\
$10-25$ & $164^{\circ} \pm 3^{\circ}$ \\
$10-10$ & $168^{\circ} \pm 2^{\circ}$ \\
planar & $163^{\circ} \pm 4^{\circ}$ \\
\hline
\end{tabular}

* The description of the electrode type indicates the target width of the linear features and the spacing between each of these features, respectively.

* The calculated mean values were obtained from six individual measurements for each representative electrode. The errors represent one standard deviation from the calculated mean values.

Similar results for the BCA values were obtained for both the patterned electrodes and the planar Ni electrodes. The average BCA for $\sim 1$-mm diameter gas bubbles was $165 \pm 2^{\circ}$. These BCA values indicate that all of the electrodes in this study exhibited a superaerophobic character, which can be attributed to their nanoscale, electrodeposited textures. ${ }^{13}$ These findings suggest that the textured Ni surfaces have a low adhesion to gas and are considered to be hydrophilic. ${ }^{14}$ These macroscale BCA measurements were, however, not able to distinguish differences in the gas wetting properties of the different microscale morphologies. These results suggest that the microscale features had little influence on macroscale gas bubbles in contrast to their electrodeposited textures. These results further suggest that the enhanced activity for the OER observed for the Ni electrodes with patterned arrays of microscale, linear features is a result of the preferential growth sites and the synchronous bubble detachment events, which were observed in the high speed video analyses. 


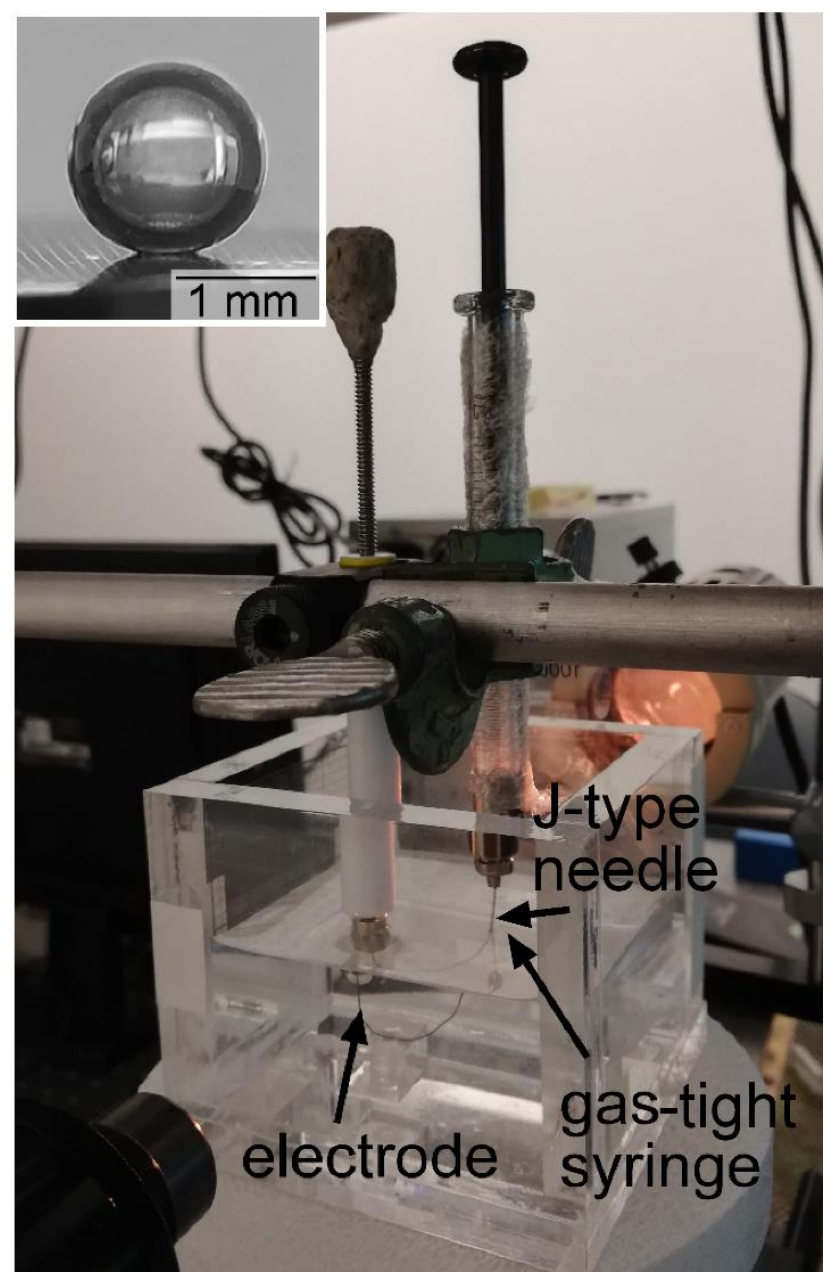

Figure S31. A photograph of the BCA set-up. The electrode is shown threaded onto a rod and placed downward into the liquid. The air bubbles were captured on the surfaces of the electrode after release from the J-type needle attached to a gas-tight syringe used for the gas injections. The inset image in the upper left-hand corner is a representative (inverted) image of a bubble captured on the surfaces of the 10-100 electrode. 


\section{References}

(1) Bedan, B.; Floner, D.; Leger, J. M.; Lamy, C. A Voltammetric Study of the Formation of Hydroxides and Oxyhydroxides on Nickel Single Crystal Electrodes in Contact with an Alkaline Solution. Surf. Sci. 1985, 162 (1-3), 822-829.

(2) Machado, S. A. S.; Avaca, L. A. The Hydrogen Evolution Reaction on Nickel Surfaces Stabilized by H-absorption. Electrochim. Acta 1994, 39 (10), 1385-1391.

(3) Grdeń, M,; Alsabet, M.; Jerkiewicz, G. Surface Science and Electrochemical Analysis of Nickel Foams. ACS Appl. Mater. \& Interfaces 2012, 4 (6), 3012-3021.

(4) van Drunen, J.; Barbosa, A. F. B.; Tremiliosi-Filho, G. The Formation of Surface Oxides on Nickel in Oxalate-Containing Alkaline Media. Electrocatalysis 2015, 6 (5), 481-491.

(5) Vogt, H.; Balzer, R. J. The Bubble Coverage of Gas-Evolving Electrodes in Stagnant Electrolytes. Electrochim. Acta 2005, 50 (10), 2073-2079.

(6) Vogt, H. The Quantities Affecting the Bubble Coverage of Gas-Evolving Electrodes. Electrochim. Acta 2017, 235, 495-499.

(7) Görlin, M.; Chernev, P.; Ferreira de Araújo, J.; Reier, T.; Dresp, S.; Paul, B.; Krähnert, R.; Dau, H.; Strasser, P. Oxygen Evolution Reaction Dynamics, Faradaic Charge Efficiency, and the Active Metal Redox States of Ni-Fe Oxide Water Splitting Electrocatalysts. J. Am. Chem. Soc. 2016, 138 (17), 5603-5614.

(8) Balej, J. Water Vapour Partial Pressures and Water Activities in Potassium and Sodium Hydroxide Solutions Over Wide Concentration and Temperature Ranges. Int. J Hydrog. Energy 1985, 10 (4), 233-243.

(9) Vogt, H. The Incremental Ohmic Resistance Caused by Bubbles Adhering to an Electrode. J. Appl. Electrochem. 1983, 13 (1), 87-88. 
(10) Vogt, H.; Thonstad, J. The Diversity and Causes of Current-Potential Behaviour at GasEvolving Electrodes. Electrochim. Acta 2017, 250, 393-398.

(11) Zhao, X.; Ren, H.; Luo, L. Gas Bubbles in Electrochemical Gas Evolution Reactions. Langmuir 2019, 35 (16), 5392-5408.

(12) Chen, Q.; Luo, L; White, H. S. Electrochemical Generation of a Hydrogen Bubble at a Recessed Platinum Nanopore Electrode. Langmuir 2015, 31 (15), 4573-4581.

(13) Hao, M.; Charbonneau, V.; Fomena, N. N.; Gaudet, J.; Bruce, D. R.; Garbarino, S.; Harrington, D. A. Guay, D. Hydrogen Bubble Templating of Fractal Ni Catalysts for Water Oxidation in Alkaline Media. ACS Appl. Energy Mater. 2019, 2 (8), 5734-5743.

(14) Darband, G. B.; Aliofkhazraei, M.; Shanmugam, S. Recent Advances in Methods and Technologies for Enhancing Bubble Detachment During Electrochemical Water Splitting. Renew. Sust. Energ. Rev. 2019, 114, 109300. 